\title{
Proton stopping measurements at low velocity in warm dense carbon
}

Sophia Malko ( $\sim$ smalko@pppl.gov )

Princeton Plasma Physics Laboratory https://orcid.org/0000-0003-1041-1092

Witold Cayzac

CEA

Valeria Ospina-Bohorquez

CEA

Krish Bhutwala

University of California San Diego

M Bailly-Grandvaux

University of California, San Diego

Christopher McGuffey

University of California, San Diego https://orcid.org/0000-0002-8162-192X

Robert Fedosejevs

University of Alberta

Xavier Vaisseau

CEA

Anna Tauschwitz

Goethe-Universitat Frankfurt am Main

Jon Apiñaniz Aginako

CLPU

Diego de Luis Blanco

CLPU

Giancarlo Gatti

CLPU

Marine Huault

CLPU

Jose Perez Hernandez

CLPU

\section{Suxing Hu}

Laboratory for Laser Energetics, University of Rochester https://orcid.org/0000-0003-2465-3818

Alexander White

Los Alamos National Laboratory

Lee Collins 
Los Alamos National Laboratory

Paul Neumayer

GSI Helmholtzzentrum für Schwerionenforschung $\mathrm{GmbH}$

\section{Gerald Faussurier}

CEA

\section{Jan Vorberger}

Institute for Radiation Physics

\section{Giuseppe Prestopino}

University of Rome https://orcid.org/0000-0002-2916-5883

\section{Claudio Verona}

Università di Roma "Tor Vergata"

\section{Joao Santos}

University of Bordeaux https://orcid.org/0000-0002-4737-8559

\section{Dimitri Batani}

Univ. Bordeaux

\section{F. Beg}

UCSD

\section{Luca Volpe}

CLPU

\section{Article}

Keywords: warm dense matter, bragg-peak region, proton energy-loss

Posted Date: September 10th, 2021

DOl: https://doi.org/10.21203/rs.3.rs-880993/v1

License: (c) (i) This work is licensed under a Creative Commons Attribution 4.0 International License. Read Full License

Version of Record: A version of this preprint was published at Nature Communications on May 24th, 2022. See the published version at https://doi.org/10.1038/s41467-022-30472-8. 


\title{
Proton stopping measurements at low velocity in warm dense carbon
}

\author{
S. Malko, ${ }^{1,2}$ W. Cayzac, ${ }^{3}$ V. Ospina-Bohórquez,${ }^{3,4,5}$ K. Bhutwala,${ }^{6}$ M. Bailly-Grandvaux,${ }^{6}$ C. McGuffey,${ }^{6,7}$ \\ R. Fedosejevs ${ }^{8}$ X. Vaisseau, ${ }^{3}$ An. Tauschwitz,${ }^{9}$ J. I. Apiñaniz, ${ }^{1}$ D. De Luis, ${ }^{1}$ G. Gatti, ${ }^{1}$ M. Huault,${ }^{1}$ \\ J. A. Perez Hernandez,${ }^{1}$ S. X. Hu, ${ }^{10}$ A. J. White, ${ }^{11}$ L. A. Collins, ${ }^{11}$ P. Neumayer, ${ }^{12}$ G. Faussurier,${ }^{3,13}$ \\ J. Vorberger ${ }^{14}$ G. Prestopino, ${ }^{15}$ C. Verona,${ }^{15}$ J. J. Santos, ${ }^{4}$ D. Batani, ${ }^{4}$ F. N. Beg, ${ }^{6}$ and L. Volpe ${ }^{1,16,17}$ \\ ${ }^{1}$ Centro de Laseres Pulsados (CLPU), Parque Cientifico, E-37185 Villamayor, Salamanca, Spain \\ ${ }^{2}$ Princeton Plasma Physics Laboratory, 100 Stellarator Road, Princeton, New Jersey 08536, United States \\ ${ }^{3}$ CEA, DAM, DIF, F-91297 Arpajon, France \\ ${ }^{4}$ University of Bordeaux, CNRS, CEA, CELIA (Centre Lasers Intenses et Applications), UMR 5107, F-33405 Talence, France \\ ${ }^{5}$ University of Salamanca, Salamanca, Spain \\ ${ }^{6}$ Center for Energy Research, University of California San Diego, La Jolla, California 92093, United States \\ ${ }^{7}$ General Atomics, San Diego, California 92121, United States \\ ${ }^{8}$ University of Alberta, Department of Electrical and Computing Engineering. Edmonton, Alberta, Canada T6G 2 V4 \\ ${ }^{9}$ Goethe-Universität Frankfurt am Main, Max-von-Laue-Strasse 1, 60438 Frankfurt am Main, Germany \\ ${ }^{10}$ Laboratory for Laser Energetics, University of Rochester, \\ 250 E. River Road, Rochester, New York 14623, United States \\ ${ }^{11}$ Theoretical Division, Los Alamos National Laboratory, Los Alamos, New Mexico 87545, United States \\ ${ }^{12}$ GSI Helmholtzzentrum für Schwerionenforschung GmbH, Planckstrasse 1, 64291 Darmstadt, Germany \\ ${ }^{13}$ Université Paris-Saclay, CEA, LMCE, F-91680 Bruyères-le-Châtel, France \\ ${ }^{14}$ Institute of Radiation Physics, Helmholtz-Zentrum Dresden-Rossendorf, \\ Bautzner Landstrasse 400, 01328 Dresden, Germany \\ ${ }^{15}$ Dipartimento di Ingegneria Industriale, Università di Roma "Tor Vergata", Via del Politecnico 1, 00133 Roma, Italy \\ ${ }^{16}$ Laser-Plasma Chair at the University of Salamanca, Salamanca, Spain \\ ${ }^{17}$ Instituto Universitario de Física Fundamental y Matemáticas, 37008-Salamanca, Spain
}

(Dated: September 6, 2021)

Ion stopping in warm dense matter is a process of fundamental importance for the understanding of the properties of dense plasmas, the realization and the interpretation of experiments involving ion-beam-heated warm dense matter samples, and for inertial confinement fusion research. The theoretical description of the ion stopping power in warm dense matter is difficult notably due to electron coupling and degeneracy, and measurements are still largely missing. In particular, the low-velocity stopping range around the Bragg peak, that features the largest modelling uncertainties, remains virtually unexplored. Here, we report proton energy-loss measurements in warm dense plasma at unprecedented low projectile velocities, approaching significantly the Bragg-peak region. Our energy-loss data, combined with a precise target characterization based on plasmaemission measurements using two independent spectroscopy diagnostics, demonstrate a significant deviation of the stopping power from classical models in this regime. In particular, we show that our results are consistent with recent firstprinciples simulations based on time-dependent density functional theory.

\section{INTRODUCTION}

Ion stopping in warm dense matter (WDM) is an important topic in inertial confinement fusion (ICF) for the ignition of small-margin ICF targets by $\alpha$-particle self-heating ${ }^{1,2}$ and for ICF schemes using ion beams as the main driver, like heavy-ion fusion ${ }^{3,4}$ or ion-driven fast ignition ${ }^{5,6}$. A precise knowledge of ion stopping in WDM is also essential for understanding proton transport in matter ${ }^{7,8}$ and for experiments where dense plasma states are generated using ion beams ${ }^{9}$, in particular proton isochoric heating ${ }^{10,11}$. Such experiments have applications for studying the structure ${ }^{12}$, the equation-ofstate $^{13}$ and the transport properties of dense plasmas ${ }^{14}$, like the conductivity ${ }^{13,15}$ and the thermal equilibration ${ }^{16}$ of WDM samples. Other applications include plasma diagnostics using ion beams ${ }^{17,18}$.

The WDM state is characterized by densities in the order or higher than the one of the solid state and temperatures below $100 \mathrm{eV}$. In this parameter range, the matter is usually partially ionized and features a significant electron coupling and electron degeneracy. These quantities are respectively measured by the non-dimensional parameters $\Gamma$ and $\Theta$, whose values for the reached conditions are approximately

$$
\Gamma=\frac{e^{2}}{a_{e} k_{B} T_{e}} \geq 0.1 \quad \text { and } \quad \Theta=\frac{k_{B} T_{e}}{E_{F}} \leq 10,
$$

where $a_{e}=\left(4 \pi n_{e} / 3\right)^{-\frac{1}{3}}$ is the average distance between the electrons, and $E_{F}$ is the Fermi energy of the free electron gas in the target. Electron coupling and degeneracy influence the electron screening properties and, thus, the plasma transport quantities including the ion stopping power $d E / d x$. 


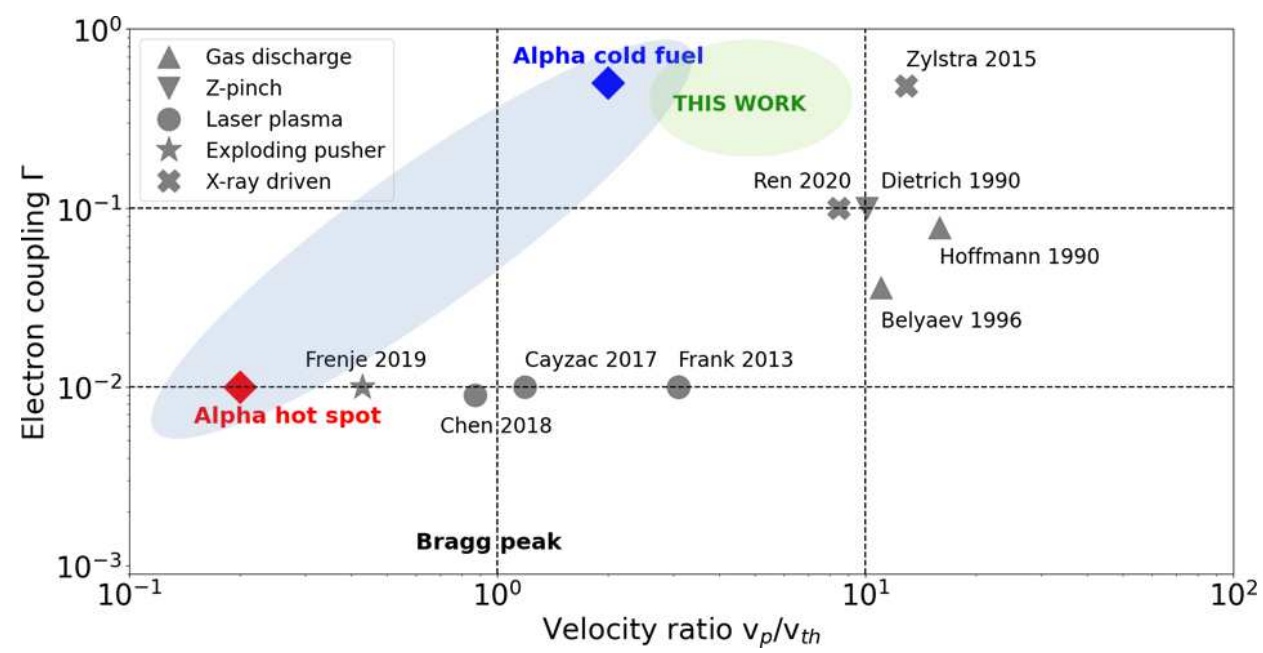

FIG. 1. Selection of reported ion-stopping experiments. Experiments displayed in the parameter space of the velocity ratio $v_{p} / v_{t h}$ of the beam-plasma interaction and the target electron coupling $\Gamma$. The grey symbols mark the plasma generation method used. The shaded blue zone represents the approximate range of $v_{p} / v_{t h}$ and $\Gamma$ values corresponding to the $\alpha$-particle emission in an igniting ICF experiment, ranging from the cold fuel to the hot spot conditions. The experiment described in this work, indicated by the shaded green zone, lies in an unexplored parameter range that is relevant for $\alpha$-particle stopping conditions in the cold fuel.

Most ion-stopping experiments have been performed in classical, highly ionized plasmas, in ideal $(\Gamma \ll 1)$ and nondegenerate $(\Theta \gg 1)$ conditions. Even in classical plasmas, measurements have chiefly been acquired at projectile velocities $v_{p}$ much larger than the thermal velocity of the plasma electrons $v_{t h}=\sqrt{\left(3 k_{B} T_{e} / m_{e}\right)}\left(v_{p}\right.$ $\left.\gg v_{t h}\right)$. In this high-velocity range, models are wellestablished and agree with experimental data ${ }^{19-22}$. In contrast, the parameter region where $v_{p} \sim v_{t h}$ (Bragg peak) is theoretically more challenging. The beamplasma coupling is here determined by binary collisions as well as interactions with density waves. Their relative and absolute contributions are strongly temperature dependent, so that even for ideal or nondegenerate conditions large discrepancies between the predictions of different stopping-power models are reported ${ }^{23,24}$. Experiments probing the Bragg peak are also more challenging, and the few measurements carried out for classical plasmas support models that include close binary collisions in the beam-plasma interaction description ${ }^{25,26}$.

For WDM target conditions, theoretical modelling is more difficult due to electron coupling and degeneracy, and requires more advanced theories like quantum manybody approaches and first-principles calculations. This leads to even larger theoretical discrepancies than in classical plasmas, in particular for low projectile velocities approaching the Bragg peak. There, temperature and degeneracy effects on the stopping power are expected to be important and significant deviations from classical theories are predicted ${ }^{27-31}$.

Measurements in WDM are also more challenging because of shorter sample lifetimes and a more difficult target characterization due to high plasma densities. A few indirect stopping measurements in degenerate conditions have been extracted from ICF implosions by using tertiary neutron spectra ${ }^{32,33}$, but these data do not allow a precise benchmarking of stopping models. The only direct ion-stopping measurements in WDM reported so far have been performed at the OMEGA laser facility ${ }^{34}$. Projectiles were quasi-monoenergetic protons of around $14.6 \mathrm{MeV}$ energy created from DHe3 fusion reactions during exploding-pusher implosions. The target was a warm dense beryllium sample isochorically heated by multi-kilojoule laser-driven X-rays over few nanoseconds, reaching $T_{e} \approx 30 \mathrm{eV}$ at solid density, corresponding to $\Gamma$ $\approx 0.3$ and $\Theta \approx 2$. However, as the beam-plasma interaction was in the high-velocity limit $\left(v_{p} / v_{t h} \approx 13\right)$, temperature and degeneracy effects on the stopping power were negligible, and the latter could be described by a simple Bethe-like formalism. Moreover, no detailed target characterization could be carried out, and only a small number of trials were able to be taken due to the scale of the laser facility. Meanwhile, lower-velocity regions approaching the Bragg peak in WDM $\left(v_{p} \sim v_{t h}\right)$ have not been experimentally investigated until now.

This is illustrated in Fig. 1, that shows a selection of reported stopping experiments displayed as a function of the velocity ratio $v_{p} / v_{t h}$ and the electron coupling parameter $\Gamma$. Experiments performed in gas-discharge and Z-pinch targets ${ }^{19-21}$ are limited to low plasma densities $\left(n_{e} \sim 10^{17-18} \mathrm{~cm}^{-3}\right)$ and the high-energy probing ion beams on the $\mathrm{MeV} / \mathrm{u}$ scale. Low to moderate velocity ratios $v_{p} / v_{t h} \leq 3$ can be obtained in laser-generated plasma and exploding-pusher experiments, which are essentially limited to hot, ideal plasmas ${ }^{22,25,26,35}$. Cold and dense plasma conditions within or approaching the WDM state can be achieved with X-ray driven plasmas ${ }^{34,36}$ but the measurements reported so far involve high velocity ratios 
$v_{p} / v_{t h} \geq 10$. Our goal is to simultaneously reach WDM states with moderate to strong electron coupling $\Gamma \sim 0.1-$ 1 and to probe them with low to moderate velocity ratio $\left(v_{p} / v_{t h} \ll 10\right)$, which remains an unexplored parameter domain and approaches the conditions of $\alpha$ projectiles in an ICF fuel shell.

Measurements at low velocity require wellcharacterized WDM samples and projectile ions with energies of a few hundred keV. Such low probing energies require thin samples which can experience a significant hydrodynamic expansion within tens to hundreds of picoseconds. Precise stopping measurements thus require a probing beam duration comparable or shorter than the sample lifetime. These requirements are difficult to achieve with accelerator ion beams because usual bunch durations lie on the nanosecond time scale. Exploding-pusher sources are limited by the relatively high $(\geq 1 \mathrm{MeV})$ reachable projectile energies and by the availability of short-duration heater beams for the WDM sample generation. On the other hand, laser-generated proton beams, that feature short pulse lengths and broadband energy spectra, offer the required flexibility to overcome these limitations. Therefore, they have been used in several recent stopping experiments $\mathrm{s}^{35,36}$ and are planned to be used in future experiments ${ }^{37,38}$, in general in association with an energy filtering device to select a narrow energy band. Moreover, as the stopping power at low velocity has a stronger temperature dependence, precise target temperature measurements are needed in order to both benchmark the plasma conditions and to interpret the energy-loss data.

In this work, we used a novel experimental approach based on a laser-generated proton selection platform operated at high-repetition rate at a short-pulse laser facility. Using this platform, we have measured, for the first time, the proton energy loss in a low-velocity regime in a warm dense carbon target that was heated by a second short-pulse laser. The projectile energy of around $500 \mathrm{keV}$ led to velocity ratios $v_{p} / v_{t h}$ between 3 and 10 , significantly lower than in previous experiments (see Fig. 1). For these conditions, discrepancies between firstprinciples stopping-power calculations and classical predictions reach up to $20 \%$ and can be resolved experimentally. Our energy-loss measurements, in association with a detailed characterization of the WDM conditions using two complementary spectroscopy diagnostics, provide a first test of ion stopping models in this unexplored regime.

\section{RESULTS}

\section{Experimental setup}

The experiment was performed at the PW-class VEGA laser facility at the Centro de Láseres Pulsados (CLPU), Salamanca, Spain ${ }^{39}$. The experimental setup is shown in Fig. 2. The initial $200 \mathrm{TW}$ VEGA2 laser beam was split into two short pulses, respectively called the main and the heater beam. The setup consists of four main stages: (i) the generation of the proton beam by the main laser beam, (ii) the generation of the WDM sample by the heater beam, (iii) the measurement of the downshifted spectrum of the proton beam that passed through the WDM target using a magnet-based spectrometer and (iv) the characterization of the WDM conditions by using two independent spectroscopy diagnostics.

The main beam, with $\approx 4 \mathrm{~J}$ energy and a $30 \mathrm{fs}$ duration, was focused onto a $3 \mu \mathrm{m}$ thick aluminium foil in order to accelerate protons through the Target Normal Sheath Acceleration (TNSA) mechanism, resulting in a broadband spectrum ${ }^{40}$ with a cut-off energy around $4 \mathrm{MeV}$. A specifically developed magnetic filtering device ${ }^{41}$ was used to select a monoenergetic pencil-like proton beam of around $500 \mathrm{keV}$ energy out of the initial spectrum to probe a target sample (solid or WDM state) located near the exit of the device. The proton beam diameter when entering the target was measured to be $50 \mu \mathrm{m}$ using radiochromic films.

The WDM sample was generated by irradiating a carbon foil of $130 \mu \mathrm{g} / \mathrm{cm}^{2}$ initial areal density, corresponding to around $1 \mu \mathrm{m}$ thickness, using the heater beam with a $0.5 \mathrm{~J}$ energy and an approximately $200 \mathrm{fs}$ duration. The heater focal spot diameter was $300 \mu \mathrm{m}$, which is much larger than the proton beam spot size and maximizes the transversal uniformity of the target conditions probed by the proton beam.

The proton beam energy spectrum was measured at high repetition rate with a magnetic spectrometer coupled with a microchannel plate (MCP) ${ }^{42}$ featuring a resolution of $2 \mathrm{keV} /$ pixel at $500 \mathrm{keV}$ energy. In this way, the optimized selected proton beam was measured to have a $498 \pm 4 \mathrm{keV}$ central energy and a $44 \pm 4 \mathrm{keV}$ energy spread. The corresponding time spread when probing the target was estimated as $400 \pm 15$ ps by using the FLUKA Monte-Carlo code ${ }^{43,44}$. The experimental proton energy loss in the target was determined by the difference between the central energies of the selected proton beam spectrum measured after free propagation in vacuum and the downshifted proton beam spectrum measured after passing through the target.

Two independent spectroscopy diagnostics were employed to characterize the WDM conditions. A Streaked Optical Pyrometry (SOP) diagnostic ${ }^{15}$ was used to determine the time-resolved black-body WDM temperature within the area probed by the protons. It detected the optical emission from the heater side of the target at $532 \mathrm{~nm}$ wavelength with a temporal resolution of around 10 ps. Simultaneously, a XUV Pinhole Grating Camera $(\mathrm{XPHG})^{45,46}$ measured the time-integrated X-ray emission in the XUV range from the WDM target heater side. The measurement was weighted over the whole heated area of around $500 \mu \mathrm{m}$ diameter and had a spectral resolution of $9 \mathrm{~nm}$. 


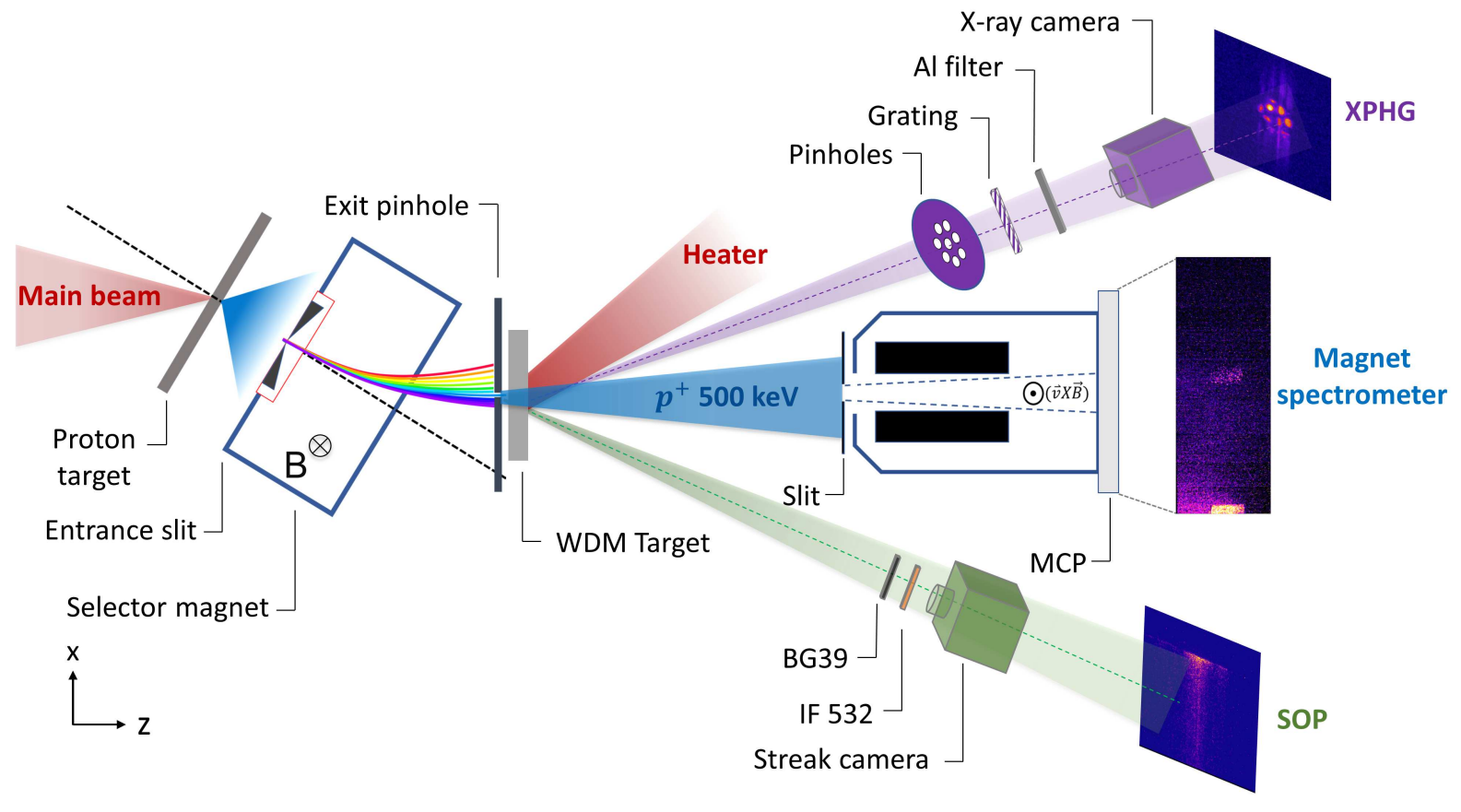

FIG. 2. Experimental setup. Scheme of the experimental setup for each shot: (i) selection of a $500 \mathrm{keV}$ energy proton beam from an initial broadband TNSA spectrum generated by the main beam, (ii) WDM sample generation by the heater beam, (iii) measurement of the downshifted proton energy spectrum of the selected beam after passing through the WDM target and (iv) characterization of the WDM sample by the SOP and the XPHG diagnostics. Typical raw experimental data acquired for each shot are shown for the magnet spectrometer as well as for the SOP and the XPHG diagnostics.

\section{Simulations of the WDM target}

The WDM conditions were simulated using the two-dimensional (2D) radiation-hydrodynamic code RALEF2D widely used for simulations of different experiments $^{47,48}$, assuming local thermodynamic equilibrium (LTE), over a 500 ps time span after the heater beam onset on the target. The target ionization is deduced by post-processing the density and temperature profiles with the LTE version of the FLYCHK code $^{50}$. The profiles of mass density $\rho$, electron temperature $T_{e}$ and mean ionization $Z^{*}$ along the target central axis are plotted in Fig. 3 for various times of the target evolution, where the target thickness is reported in areal-density units. The reached conditions are $\rho \geq 0.1 \mathrm{~g} / \mathrm{cm}^{3}$ and $T_{e}$ between a few $\mathrm{eV}$ and a few tens of $\mathrm{eV}$, which correspond to carbon ionization degrees $Z^{*} \leq 4$. The resulting values of $\Gamma \approx 0.1-2$ and $\Theta \leq 10$ (in most of the target) are also shown in Fig. 3, indicating moderately to strongly coupled, and moderately degenerate target conditions. The velocity ratio values corresponding to the projectile energy of $500 \mathrm{keV}$ are also plotted, with $v_{p} / v_{t h} \leq 10$ over the considered time domain, and $v_{p} / v_{t h} \approx 2-3$ in the first tens of picoseconds of the target evolution, which is significantly lower than in previous experiments. As also appears on the graphs of Fig. 3, the target areal density remains remarkably constant, which indicates a one-dimensional target expansion over the time range of
500 ps.

\section{WDM target characterization}

The temporal evolution of the target temperature extracted from the SOP data averaged over 80 shots is shown in Fig. 4 a) and compared with the time-dependent temperature extracted from the RALEF2D simulation. Both the experimental and the simulated temperature are determined at the critical density for the $532 \mathrm{~nm}$ wavelength used for the measurements and averaged within a $50 \mu \mathrm{m}$ emission diameter around the central plasma axis corresponding to the proton beam probing area. The experimental error results from the statistical error on the measurements and from the detector calibration uncertainty and is estimated as $\pm 30 \%$. The error band on the simulation curve accounts for the signal variation due to shot-to-shot pointing fluctuations of the heater beam estimated to be below $50 \mu \mathrm{m}$. The temperature determined from the SOP data is slightly lower than the one predicted by the RALEF2D simulation, while agreeing, in average, within the $\pm 30 \%$ experimental error bar. The experimental temperature is also compared to the temperature extracted from a hydrodynamic simulation performed with the one-dimensional (1D) MULTI-fs code ${ }^{51}$ in LTE. The MULTI-fs prediction, also determined at the critical density for the $532 \mathrm{~nm}$ 

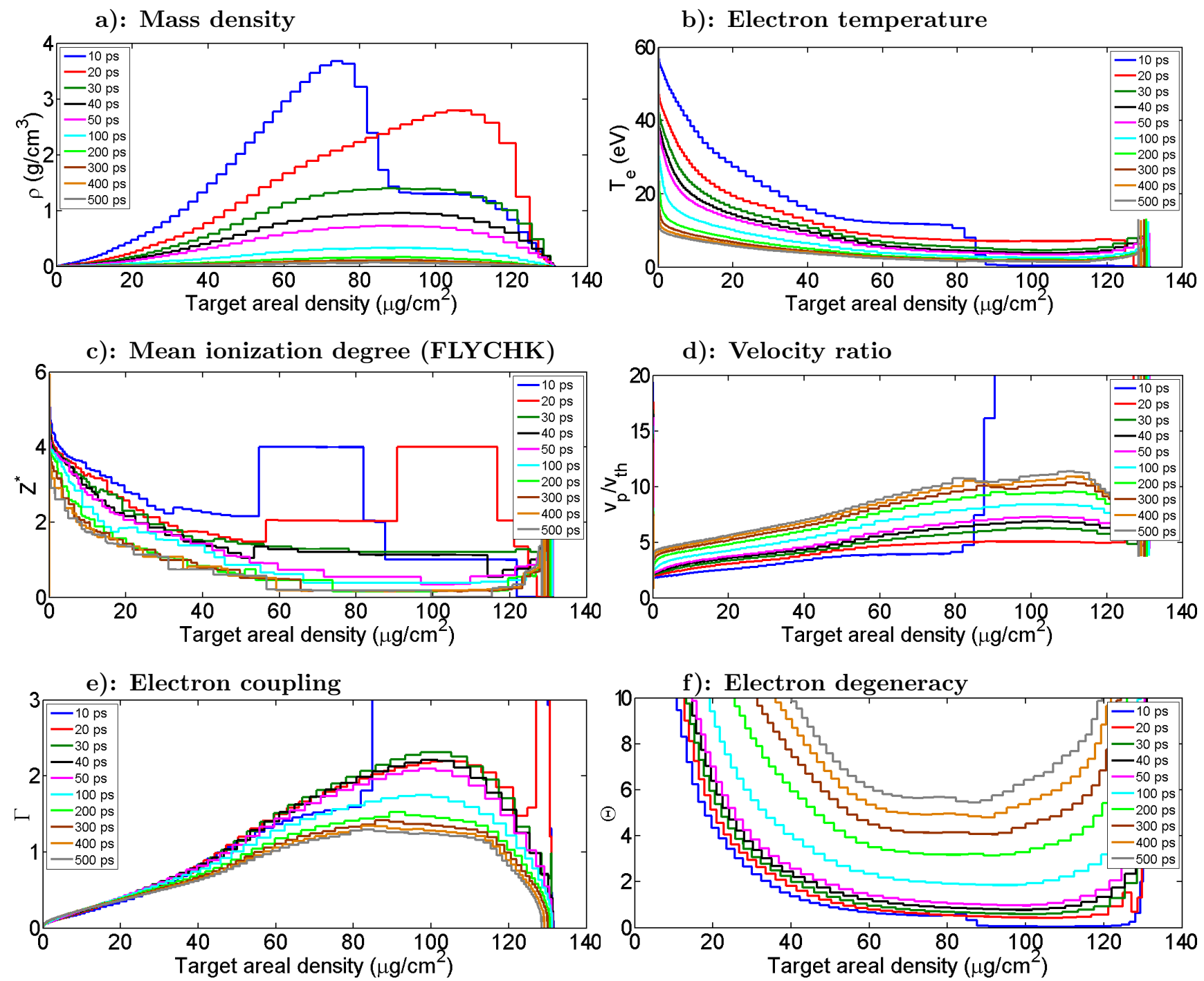

FIG. 3. RALEF2D hydrodynamic simulation. Target profiles along the plasma central axis for $t=0-500 \mathrm{ps}$ after the beginning of the laser heating. a) Mass-density. b) Electron temperature. c) Mean ionization calculated with the FLYCHK code at LTE. Discontinuities at early times are a calculation artefact. d) Velocity ratio $v_{p} / v_{t h}$ for $500 \mathrm{keV}$ energy projectiles. e) Electron coupling $\Gamma$. f) Electron degeneracy $\Theta$. The x-axis is reported in areal-density units $\left(\mu \mathrm{g} / \mathrm{cm}^{2}\right)$.

wavelength, overestimates the measured temperatures by around $30 \%$. The RALEF2D prediction is clearly more accurate as the simulation was performed using the experimentally measured spatial distribution of the heater focal spot intensity.

The X-ray emission spectra over the whole target emission area measured with the XPHG diagnostic are presented in Fig. $4 \mathrm{~b}$ ). They are compared to the spatially and temporally integrated spectra obtained with the PrismSPECT code $^{52,53}$ assuming LTE and using the density and temperature profiles extracted from both the RALEF2D simulation (Fig. $3 \mathrm{a}$ ) and b) respectively) and from the MULTI-fs 1D hydrodynamic simulation carried out over a weighted range of intensities matching the experimentally measured focal spot. The measured spectra agree within 10-30\% with the spectra predicted by the RALEF2D and the MULTI-fs codes, which is on the order of the experimental error bar estimated as $\approx 20 \%$. In contrast to the SOP data, the XPHG measurement shows an X-ray emission at higher energies than simulated, which corresponds to an experimental temperature higher than simulated.

Based on the RALEF2D simulation, a mass-weighted and time-integrated temperature of $7.5 \mathrm{eV}$ is estimated within the $50 \mu \mathrm{m}$ proton diameter spot. Taking the average of both diagnostics, it can be concluded that the measured temperature is within $15 \%$ agreement with the RALEF2D simulation. The good agreement of the XPHG data with the RALEF2D prediction also shows that the target electron density is known with a reason- 
a): SOP averaged temperature

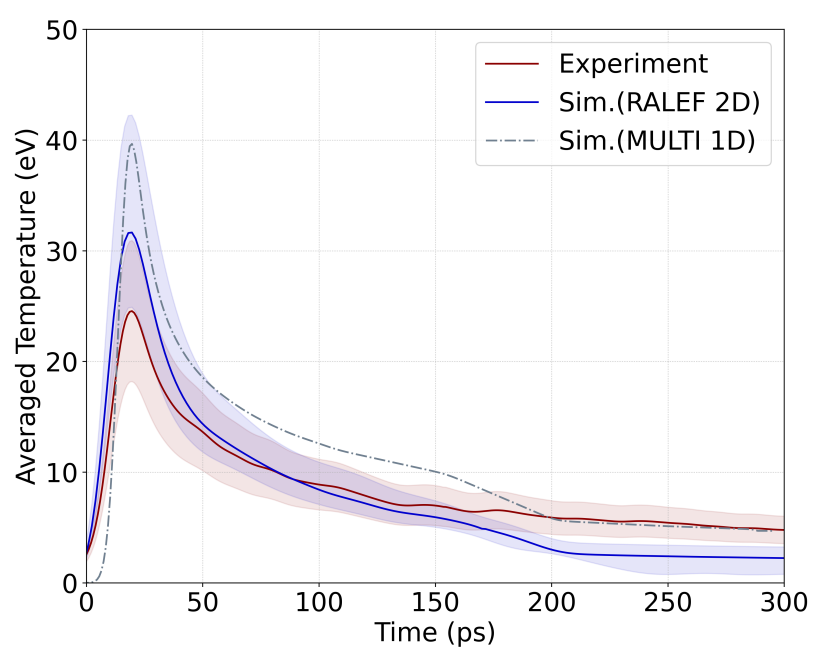

b): X-ray emission spectra

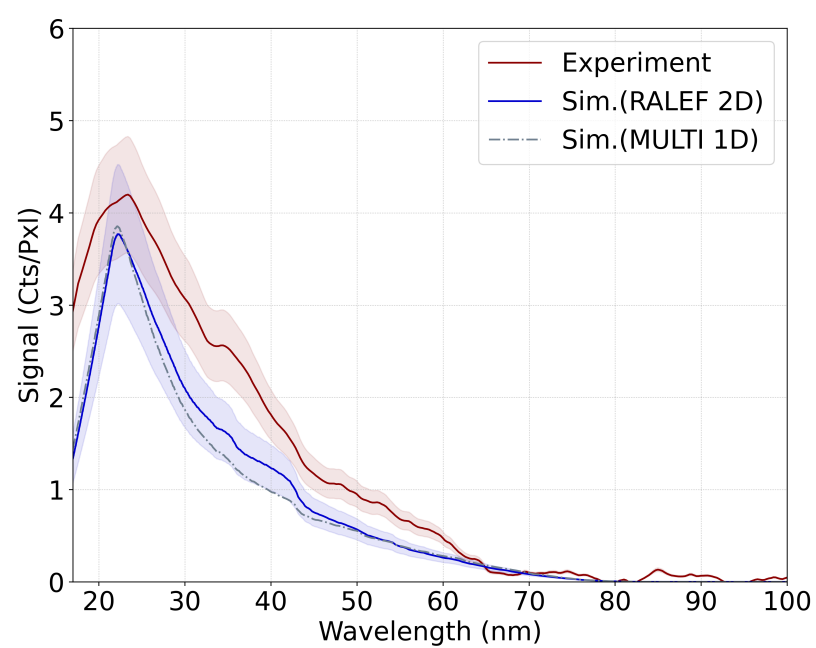

FIG. 4. WDM Characterization. a) Streaked Optical Pyrometry (SOP) measurement. Temperature evolution as a function of time (red curve) averaged within the $50 \mu \mathrm{m}$ diameter proton probing area compared with the temperatures extracted from the 2D RALEF2D (blue curve) and the 1D MULTI-fs (dashed grey curve) hydrodynamic codes, determined at the critical density for a $532 \mathrm{~nm}$ wavelength. b) X-ray pinhole grating camera (XPHG) measurement. Experimental time-integrated X-ray emission (red curve) compared with the prediction obtained with the PrismSPECT code by post-processing the hydrodynamic profiles obtained with the RALEF2D (blue curve) and MULTI-fs (dashed grey curve) hydrodynamic codes. The simulation curves are convoluted with the respective resolutions of $10 \mathrm{ps}$ for the SOP diagnostic and $15 \mathrm{~nm}$ for the XPHG diagnostic.

able accuracy. Moreover, the overall agreement of both measurements with the simulation over the whole considered time range indicates that the target expansion and thus the target areal density, are correctly simulated. In particular, the agreement of the experimental data with both the $2 \mathrm{D}$ and the $1 \mathrm{D}$ hydrodynamic simulations confirms that the target expansion is nearly one-dimensional as predicted by the RALEF2D code. Therefore, the presented spectroscopy measurement data set enables to validate the target parameters over the time domain of interest of a few hundred of ps. It is worth mentioning that this WDM sample characterization has been carried out simultaneously with the proton energy-loss measurements, which has not been done in previous stoppingpower experiments.

\section{Stopping-power calculations}

For estimating the discrepancies between stoppingpower models for typical conditions of the experiment, various predictions for protons in carbon are compared in Fig. 5a for a density $\rho=0.5 \mathrm{~g} / \mathrm{cm}^{3}$ and a temperature $T_{e}=10 \mathrm{eV}$. The proton stopping power in solid carbon according to the SRIM database ${ }^{54}$ is plotted as a reference.

Firstly, we use ad hoc calculations combining a freeelectron and a bound-electron contribution that are obtained separately knowing the target ionization ${ }^{55-57}$. The free-electron term is calculated using several mod- els that have the same Bethe-like high-velocity limit determined from dielectric stopping theory: the LiPetrasso (LP) model $^{58}$, the Brown-Preston-Singleton (BPS) model ${ }^{59}$, the T-matrix (TM) model with velocitydependent screening 23,60 , the dielectric random phase approximation (RPA) model ${ }^{23,61}$ and the Zimmerman parametrization $^{62}$ of the Maynard-Deutsch dielectric stopping power ${ }^{63}$, the latter being very similar to the RPA description. In all cases, the bound-electron stopping term, which is specifically plotted in Fig. 5a and Fig. 5b, is calculated using a model by Casas et al. ${ }^{56}$ that is valid for all projectile velocities.

Secondly, we use a self-consistent average-atom method in the local density approximation that simultaneously calculates the plasma ionization and the total stopping power using the method presented in Ref. ${ }^{64,65}$ and using the quantum average atomic model (QAAM) described in Ref. ${ }^{66}$ under the LTE assumption.

Thirdly, we employ an ab initio approach based on a recently developed time-dependent density functional theory (TD-DFT), including an orbital-free (TD-OFDFT) version ${ }^{30,31}$ and a full Kohn-Sham approach (TDKS-DFT) utilizing a mixed basis of deterministic and stochastic orbitals $^{67}$. It has been shown in Ref. ${ }^{30,31}$ that the TD-OF-DFT theory agrees with the high-velocity data of Ref. ${ }^{34}$, but predicts deviations of up to $20 \%$ from classical stopping-power predictions for WDM conditions at low projectile velocities. The TD-OF-DFT and TDKS-DFT values are determined with an uncertainty estimated to $\pm 10 \%$. 
The ad hoc and the QAAM calculations are in close agreement for proton energies $E_{p} \geq 500 \mathrm{keV}$ and predict a significant increase of the stopping power compared to the solid, that reaches $\approx 20 \%$ at $E_{p}=500 \mathrm{keV}$. Discrepancies between these models increase at lower energies. In contrast, the TD-OF-DFT and the TD-KS-DFT theories predict a stopping power close to or slightly below the solid level for $E_{p} \geq 400-500 \mathrm{keV}$. The more precise TDKS-DFT predictions are smaller and within better than $10 \%$ agreement with the TD-OF-DFT values. A stopping enhancement relative to the solid is also predicted but at lower velocities than according to other calculations, in the close vicinity of the Bragg peak. Hence, in the probing range of $500 \mathrm{keV}$ energy, a $20 \%$ reduction of the stopping power is predicted by the TD-OF-DFT and TD-KS-DFT theories compared to the other models, which we attribute to the electron coupling and quantum degeneracy more precisely included in the TD-DFT calculations.

The effect of the target temperature on the stopping power is shown in Fig. 5b for an ad hoc calculation (namely with the Zimmerman model), and for the QAAM and TD-OF-DFT models, respectively, for the same density $\rho=0.5 \mathrm{~g} / \mathrm{cm}^{3}$ and for temperatures $T_{e}=$ 10, 20, and $30 \mathrm{eV}$. For these conditions, the ionization degree according to FLYCHK is $Z^{*}=1.43,2.31$ and 2.95 , while the one estimated with QAAM is $Z^{*}=1.56$, 2.40 and 2.86 , respectively. As is shown, the stoppingpower variation with temperature is very small for proton energies above $200-300 \mathrm{keV}$, reaching few percent at the experimental projectile energy of $500 \mathrm{keV}$. This also shows that the variation of the ionization degree with temperature on the one hand and the small ionization differences used for the various stopping-power calculations on the other hand are negligible for the studied conditions. These estimates thus suggest that the stopping power in the studied beam-target parameter range is not impacted by thermal effects, but that it is significantly impacted by coupling and/or degeneracy effects as shown by the discrepancy between the first-principle TD-OF-DFT calculation on the one hand and the classical and average-atom calculations on the other hand. For comparison with the experimental measurements, we calculated the energy loss $\Delta E_{\text {sim }}$ at each time step of the hydrodynamic simulation as the integral of the stopping power along the ion trajectory through the target

$$
\Delta E_{\operatorname{sim}}=-\int \frac{\partial E}{[\rho(x) \partial x]}[\rho(x) d x]
$$

where the stopping power, expressed as an energy loss per unit of areal density, is calculated with the parameter profiles as shown in Fig. 3. Each energy-loss value is averaged over the target parameters in a temporal range of $400 \mathrm{ps}$ corresponding to the duration of the proton bunch interacting with the target, as well as in a spatial range of $50 \mu \mathrm{m}$ corresponding to the probing proton beam diameter. The calculation was respectively performed
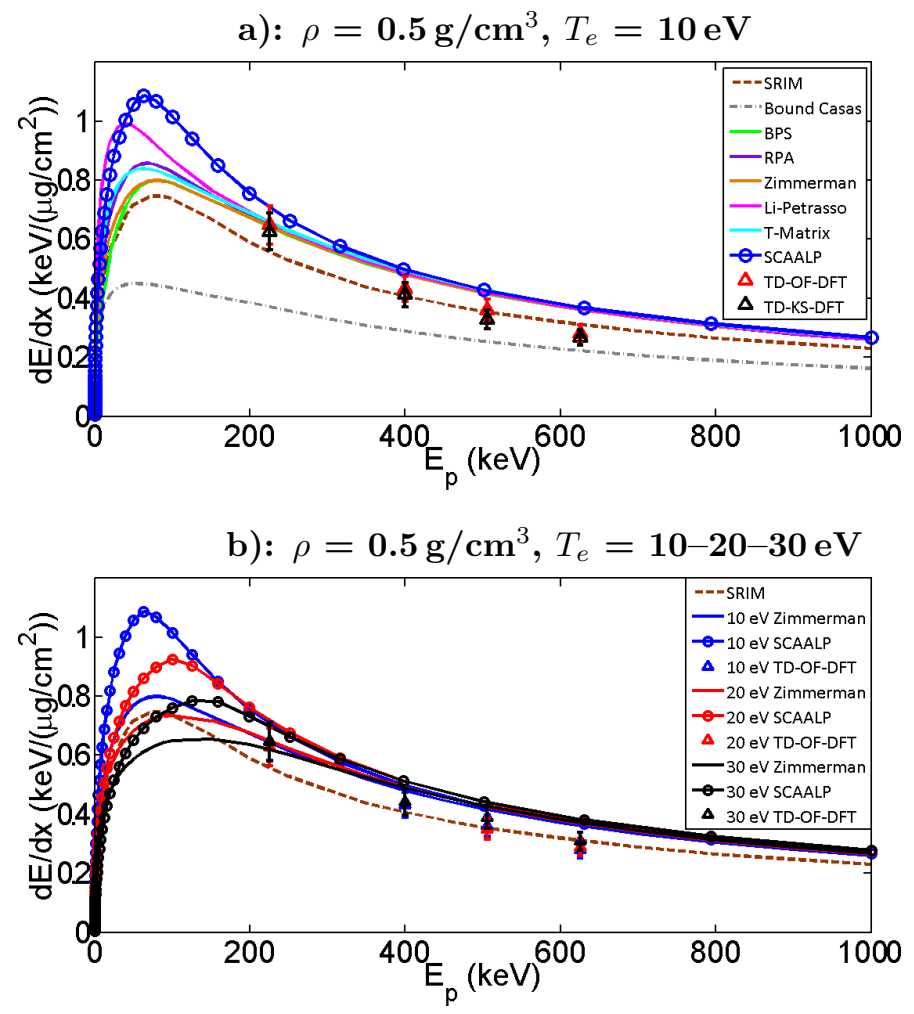

FIG. 5. Comparisons of proton stopping power in warm dense carbon. a) Stopping power for $\rho=0.5 \mathrm{~g} / \mathrm{cm}^{3}$ and $T_{e}=10 \mathrm{eV}$. b) Stopping power for $\rho=0.5 \mathrm{~g} / \mathrm{cm}^{3}$ and various temperature values $T_{e}=10 \mathrm{eV}, 20 \mathrm{eV}$ and $30 \mathrm{eV}$.

for the cases where the proton beam is centered on the target central axis and where the proton beam is deviated by $50 \mu \mathrm{m}$ from the central axis, which corresponds to the maximum estimated pointing fluctuation between the proton and the heater beam in the experiment.

For computational effectiveness, the energy loss is only calculated in an ad hoc manner, using the Zimmerman, Li-Petrasso and T-Matrix models for the free-electron stopping and the Casas model for the bound-electron stopping. These three calculations predict very similar values within $1 \%$ (as also suggested by Fig. 5), and are simply designated as "classical calculation" in the following. Due to the low sensitivity of the stopping power to temperature in the studied conditions, the experimental uncertainty of $\pm 15 \%$ on the $T_{e}$ measurement is neglected in the energy-loss calculation.

\section{Energy-loss results}

Firstly, the energy loss of the proton beam was measured in solid carbon foils over 35 shots to estimate the measurement accuracy and provide a reference energyloss value in the solid target $\Delta E_{\mathrm{sol}}$. The downshifted proton energy after passing the solid target was measured to be $449 \pm 5 \mathrm{keV}$, where the error $\sigma= \pm 5 \mathrm{keV}$ results 
from the standard deviation at $1 \sigma$ over all shots and from systematic measurement uncertainties as is explained in the methods. This results in an energy loss of $\Delta E_{\mathrm{sol}}=$ $49 \pm 5 \mathrm{keV}$, which is in good agreement with the energy loss of $48.1 \mathrm{keV}$ predicted with the SRIM database ${ }^{54}$.

Subsequently, the proton energy loss in the sample was measured on shots with the heater beam driving the target, at respective time delays of $-316 \pm 100 \mathrm{ps},-116 \pm$ $100 \mathrm{ps}$ and $86 \pm 100 \mathrm{ps}$ relative to the onset of the heater laser pulse on the sample. The experimental data acquired over several shots are presented at each time delay in Fig. 6 a), b) and c), where each data point corresponds to an individual shot. The blue band on each graph corresponds to the experimental error interval of $\pm 5 \mathrm{keV}$ of the reference energy-loss measurement in the solid target $\Delta E_{\mathrm{sol}}$.

In Fig. 6 a) and b), the energy loss is measured before the laser heating of the sample, for protons still probing the solid target. The obtained data points are consistent with the previous reference energy-loss measurement in the solid foil $\Delta E_{\mathrm{sol}}$.

In contrast, in Fig. 6 c), the energy-loss measurement is performed when the temporal center of the proton beam is at $86 \mathrm{ps}$ after the beginning of the sample heating, so that protons almost fully probe the WDM state. The measured energy loss reaches values between $36 \pm 5 \mathrm{keV}$ and $43 \pm 5 \mathrm{keV}$ depending on the shot, with an average value $\Delta E_{\mathrm{WDM}}$ of $39.4 \pm 5 \mathrm{keV}$ over four shots. This corresponds to values of $13-26 \%$ lower than the measurement in the solid target $\Delta E_{\mathrm{sol}}$ of $49 \mathrm{keV} \pm 5 \mathrm{keV}$, with an average percentage difference of $20 \pm 9 \%$.

A comparison of the averaged proton spectra acquired respectively after free propagation in vacuum, after passing the solid target and after passing the WDM target is shown in Fig. 6 d). The clear shift in the central proton energy between the spectra in the solid and in the WDM target indicates a reduced energy loss $\Delta E_{\mathrm{WDM}}$ in the WDM state.

The experimental data in Fig. 6 a),b),c) are compared with the results of the classical energy-loss calculation $\Delta E_{\text {sim. }}$. The calculation result assuming a $50 \mu \mathrm{m}$ offset between the proton and the heater beam is slightly lower, by $1-2 \mathrm{keV}$, than the one along the central target axis due to lower temperatures of the probed region. At the time of proton probing in WDM, the calculated values $\Delta E_{\text {sim }}$ are respectively $55.3 \mathrm{keV}$ and $54 \mathrm{keV}$. These values are $15 \%$ and $12 \%$ higher than the SRIM energy loss in the solid target and $12 \%$ and $10 \%$ higher that the measured energy loss in the solid $\Delta E_{\text {sol }}$. Hence, the energy loss measured in the WDM sample, with an average value $\Delta E_{\mathrm{WDM}}=39 \pm 5 \mathrm{keV}$, is at least $15 \mathrm{keV}$ lower than the classical prediction. These differences are greater than the error bars and thus suggest that the classical calculation $\Delta E_{\text {sim }}$ overestimates the measured energy loss by $41 \%$.

In contrast, as shown in Fig. 5, the stopping power predicted by TD-OF-DFT and TD-KS-DFT calculations at $500 \mathrm{keV}$ projectile energy for typical temperatures of the experiment is close or even smaller than the stopping power in the solid. In particular, for the case of Fig. 5a) with $T_{e}=10 \mathrm{eV}$ which is close to the experimental measurement, the TD-KS-DFT calculation predicts a $\approx 8 \%$ reduced proton stopping power compared to the one in the solid. Therefore, the trend of our energy-loss measurements that indicates a reduced stopping power in WDM is consistent with the TD-DFT stopping-power predictions. However, all other models that predict a stopping-power enhancement for the considered WDM conditions are not consistent with our data and appear to be not valid in the probed parameter range.

\section{Conclusions}

In summary, our proton energy-loss data at $500 \mathrm{keV}$ energy in warm dense carbon, at a velocity ratio down to $v_{p} / v_{t h} \geq 3$, provide a first measurement in the unexplored regime of low-velocity stopping in coupled and degenerate plasma conditions.

These results, when compared with existing stoppingpower models, suggest a general agreement with the Density Functional Theory (TD-OF-DFT and TD-KS-DFT) calculations, which predict a stopping power similar or slightly reduced with respect to the one in the solid target. At this projectile energy, these theoretical approaches are the only ones that are consistent with our measurements. In contrast, the ad hoc and average-atom models significantly overestimate the stopping power in this regime. This highlights the effect of electron coupling and degeneracy at low projectile energy in WDM, which reduces the stopping power compared to classical approaches. This result has strong implications for experiments where the energy loss of ions in WDM plays a significant role, where classical stopping-power modelling is usually employed. It thus calls for the use of more detailed calculations in this stopping regime based on first-principles methods like the Density Functional Theory.

Moreover, our plasma emission measurements carried out using the SOP and XPHG spectroscopy diagnostics simultaneously to the stopping measurements, provide a WDM target characterization never achieved, to our knowledge, in previous stopping experiments. In particular, the experimentally determined temperature is in agreement within $20 \%$ with hydrodynamic simulations. This confirms the sample probing within the interesting regime of intermediate coupling $(\Gamma \sim 1-2)$ and degeneracy $(\Theta \leq 4)$.

Several developments of this experimental approach are possible by further refining the experimental parameters for increasing the precision and the accuracy of our measurements in order to provide more accurate comparisons with the theories. The proton energy selector can be optimized to reduce the proton beam bandwidth and time spread, with the goal of achieving a $5 \%$ measurement accuracy in the future. A proton focusing system 
a)

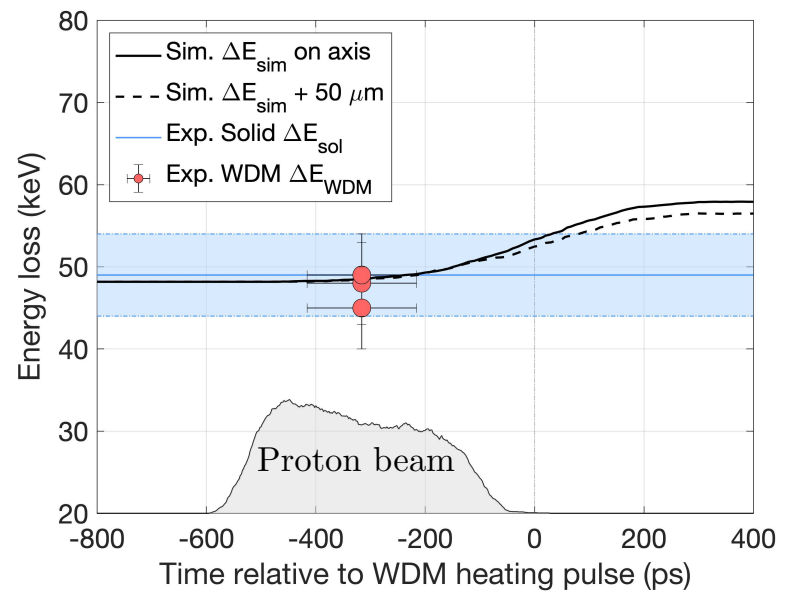

c)

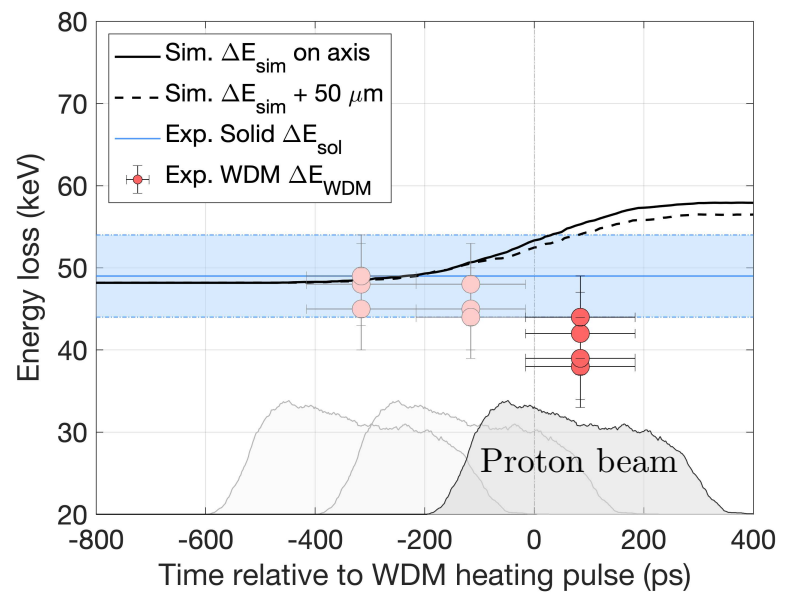

b)

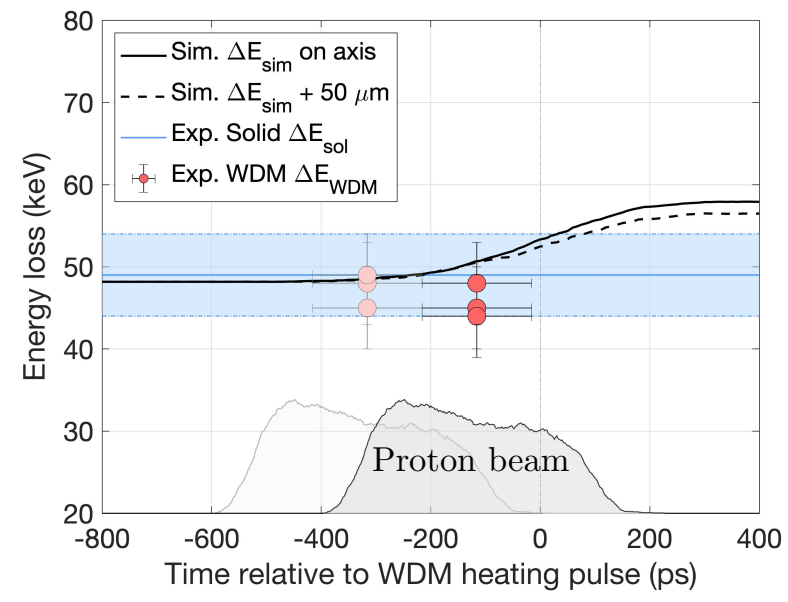

d)

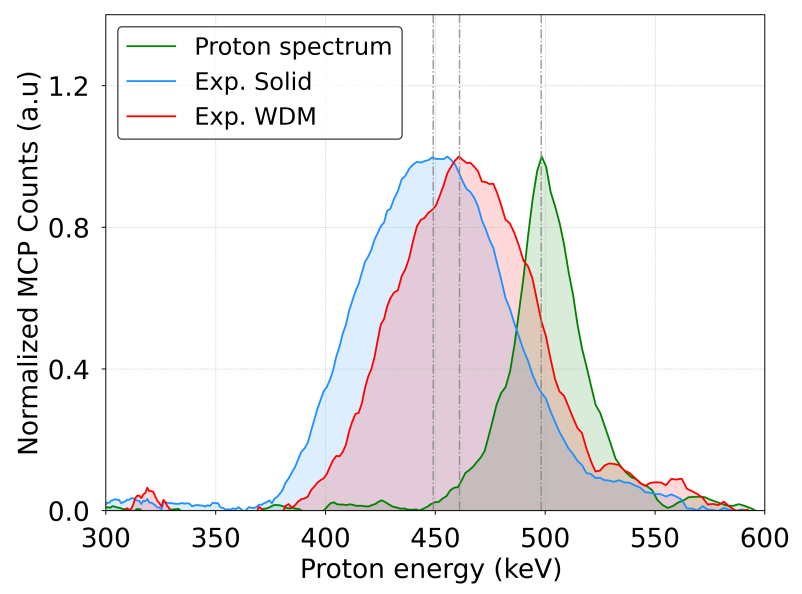

FIG. 6. Experimental energy-loss results. Proton energy-loss data as a function of time. The time $t=0$ ps corresponds to the onset of the heater beam on the carbon target. The temporal shape of the probing proton bunch obtained with a FLUKA Monte-Carlo simulation is represented as a shaded grey profile. The averaged measured energy loss in the solid target $\Delta E_{\text {sol }}$ is plotted as a blue solid line, and the surrounding light blue band indicates the error $\sigma= \pm 5 \mathrm{keV}$. The black solid and dashed curves represent the results of the classical energy-loss calculation assuming the proton beam probing the target along its central axis and with a $50 \mu \mathrm{m}$ offset, respectively. a) Energy-loss measurement at $t=316 \pm 100 \mathrm{ps}$ prior to the heater pulse onset. b) Energy-loss measurement at $t=116 \pm 100 \mathrm{ps}$ prior to the heater pulse onset. c) Energy-loss measurement at $t=86 \pm 100 \mathrm{ps}$ after the heater pulse onset. d) Comparison of the proton spectrum measured after free propagation in vacuum (averaged over 20 shots, green curve), the downshifted proton spectrum after passing the solid target (averaged over 35 shots, blue curve), and the downshifted proton spectrum after passing the WDM target (averaged over 4 shots, red curve). The vertical bars mark the spectra maxima positions.

can also be set up after the WDM target to mitigate the effects of angular straggling and maximize the proton collection, increasing the measurement precision by about $50 \%$. Furthermore, the selector can be adjusted to investigate various projectile energy ranges between $100 \mathrm{keV}$ and $2 \mathrm{MeV}$. Energies of $100-200 \mathrm{keV}$ are of particular interest to investigate the regime of $v_{p} / v_{t h}=1-2$ near the Bragg Peak, where the largest theoretical discrepancies are reported.

\section{METHODS}

\section{Experimental}

\section{Lasers and targets}

The initial $4 \mathrm{~J}$ energy, 30 fs duration and $200 \mathrm{TW}$ power VEGA 2 beam was split into two beams by using a $90 \%$ reflecting beam splitter ${ }^{39}$.

The main beam, that contains $90 \%$ of the total energy, was used to accelerate protons via the Target Normal 
Sheath Acceleration (TNSA) mechanism. It was focused using an $F / 13(F=130 \mathrm{~cm})$ parabolic mirror onto a $3 \mu \mathrm{m}$ thick aluminium foil at a $14.5^{\circ}$ incidence angle. The pulse duration was $30 \mathrm{fs}$ and the focal spot diameter was $20 \mu \mathrm{m}$ at full width at half maximum (FWHM), yielding an intensity on target of $\sim 10^{19} \mathrm{~W} / \mathrm{cm}^{2}$. The aluminium foils had dimensions of $\approx 80 \times 80 \mathrm{~mm}$ and were mounted in a motorized sandwich holder with a matrix of $45 \times$ 45 apertures of $800 \mu \mathrm{m}$ diameter each. Each aperture was used for one shot, which allowed a quick shot-to-shot target switch for operation at high repetition rate.

The heater beam containing the remaining $10 \%$ laser energy was stretched to a $217 \mathrm{fs}$ pulse duration that was measured using a second-order autocorrelator with an accuracy of 5 fs. For generating the WDM sample, the beam was focused onto a carbon target with an incidence angle of $35^{\circ}$ and a focal spot diameter of $300 \mu \mathrm{m}$, which yielded an intensity on target $\sim 10^{16} \mathrm{~W} / \mathrm{cm}^{2}$. The carbon target was positioned at a $0.9 \mathrm{~cm}$ distance from the exit pinhole of the selector in the proton propagation axis and at $\mathrm{a} \sim 8 \mathrm{~cm}$ distance from the proton source point.

The targets were portions of large $80 \mathrm{~mm}$ diameter carbon foils that were manufactured by resistance evaporation under high vacuum ${ }^{68}$ at the GSI Target Laboratory. The energy-loss measurements in the solid and in the WDM target presented in this work were performed with the same carbon foil with an average initial areal density of $130 \mu \mathrm{g} / \mathrm{cm}^{2}$ with an uncertainty of $\pm 1 \%$. With a carbon density $\approx 1.3 \mathrm{~g} / \mathrm{cm}^{3}$, this areal density value corresponds to a $1 \mu \mathrm{m}$ initial target thickness. For allowing measurements at high repetition rate, the foils were mounted in a holder with a matrix of $45 \times 45$ apertures, each aperture corresponding to one target used on one shot. The aperture diameter was of $1 \mathrm{~mm}$ on the proton incidence side and of $800 \mu \mathrm{m}$ on the heater-beam side, which was designed to ensure the integrity of neighbouring targets during each shot because of the high fragility of the foil. The good agreement of the measurements in solid carbon with the SRIM prediction for a $130 \mu \mathrm{g} / \mathrm{cm}^{2}$ areal density shows that possible effects of areal-density variations across the foil surface are within the experimental energy-loss error bars and do not impact the data analysis.

\section{Magnet Spectrometer}

The magnet spectrometer was designed and characterized at CLPU and was used to measure the proton beam energy. It was positioned at a $38.2 \mathrm{~cm}$ distance from the WDM sample along the proton propagation axis. The spectrometer consists of a $0.2 \mathrm{~T}, 10.4 \mathrm{~cm}$ long dipole magnet. It deflects protons upwards to a microchannel plate (MCP) detector, which is coupled with a phosphor screen located $10 \mathrm{~cm}$ from the end of the magnet and imaged onto a CCD camera. The 2D magnetic field of the spectrometer was measured with a Hall effect probe and was used to calculate the predicted proton deflection on the
MCP. The resolution of the spectrometer at $500 \mathrm{keV}$ proton energy is $2 \mathrm{keV}$ per pixel of the image. A horizontal slit of $1 \mathrm{~mm}$ height and $1 \mathrm{~cm}$ length was inserted in front of the spectrometer entrance aperture of $1 \mathrm{~cm}$ diameter to ensure that only protons within the horizontal plane of the propagation axis enter the spectrometer. This provides the "zero height" (zero deflection) reference position on the detector. The vertical positioning uncertainty of the proton beam of $\pm 125 \mu \mathrm{m}$ results in an energy uncertainty of $\pm 2.5 \mathrm{keV}$ on the MCP that constitutes a systematic error $\sigma_{\mathrm{sys}_{1}}$ on the energy measurement.

Examples of raw images obtained with the MCP detector for individual shots are presented in Fig. 7, which shows a reference signal of the selected proton beam (a)) and selected proton beam signals after passing through target samples (b) - d)). As is visible in Fig. 7 b), the angular straggling of the proton beam through the target , which is estimated to be around $2^{\circ}$ using FLUKA simulations, results in a $3 \mathrm{~cm}$ spot at the spectrometer entrance. This signal broadening introduces an error in the estimation of the central energy of the downshifted proton spectrum. In order to mitigate this error, we mounted a horizontal slit of $1 \mathrm{~mm}$ height in front of the spectrometer for reducing the beam spot size on the MCP detector as illustrated in Fig. 7 c). Using this slit, a systematic error is added on the energy measurement in the sample due to partial collection of protons on the detector, which is estimated as $\sigma_{\text {sys }_{2}}= \pm 3.5 \mathrm{keV}$.

The total error on the energy-loss measurement is estimated as $\sigma_{\text {tot }}=\sqrt{\sigma_{\text {stat }}^{2}+\sigma_{\text {sys }_{1}}^{2}+\sigma_{\text {sys }_{2}}^{2}}$, where $\sigma_{\text {stat }}^{2}=$ $\sigma^{2} / N$. Here, $\sigma$ is the standard deviation and $N$ is the number of shots, while $\sigma_{\mathrm{sys}_{1}}= \pm 2.5 \mathrm{keV}$ and $\sigma_{\mathrm{sys}_{2}}= \pm$ $3.5 \mathrm{keV}$ are the systematic errors coming respectively from the proton beam alignment and from the aforementioned partial collection of protons. The energy loss in the target is estimated as the difference between the measured central energies of the reference and of the downshifted beam, and can be written as $\Delta E_{\text {down }}=E_{\text {ref }}$ $\left(\sigma_{\text {stat }}, \sigma_{\mathrm{sys}_{1}}\right)-E_{\text {down }}\left(\sigma_{\text {stat }_{1}}, \sigma_{\mathrm{sys}_{1}}, \sigma_{\mathrm{sys}_{2}}\right)$.

\section{Energy selector}

The energy selector was designed and characterized at CLPU as a compact adjustable platform for proton stopping-power measurements with working range energies of up to a few $\mathrm{MeV}$. It is $\sim 6 \mathrm{~cm}$ long and it consists of a $1.2 \mathrm{~T}$ permanent dipole magnet that deflects protons in the horizontal plane using two apertures. The first one is placed at the magnet entrance (entrance slit) and the second one at the magnet exit (exit pinhole). The selector is positioned at $1.6 \mathrm{~cm}$ from the proton source and it is rotated by $14.5^{\circ}$ for pointing the selected proton beam in straight axis with respect to the WDM sample. The entrance slit, of $20 \mu \mathrm{m}$ width and $3 \mathrm{~mm}$ height, is attached in front of the dipole magnet yoke for reducing the horizontal acceptance of the incoming TNSA proton 


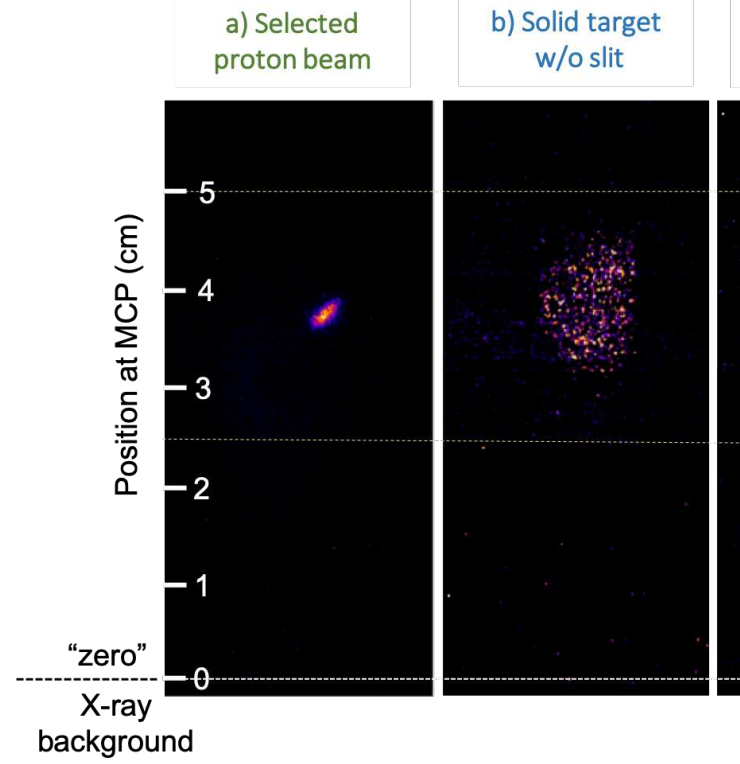

c) Solid target

w/ slit
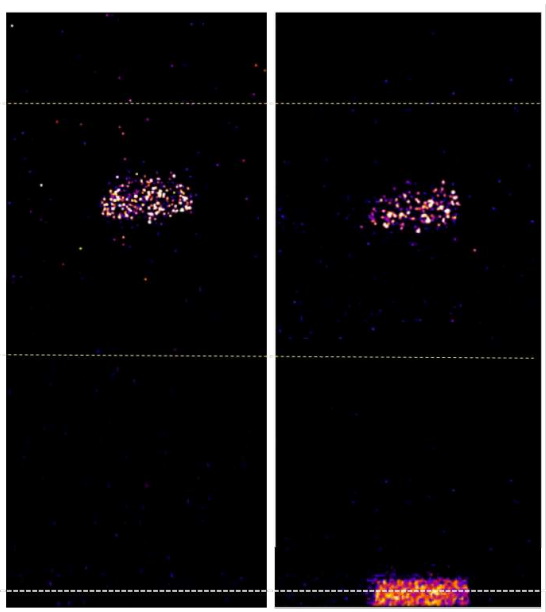

e)

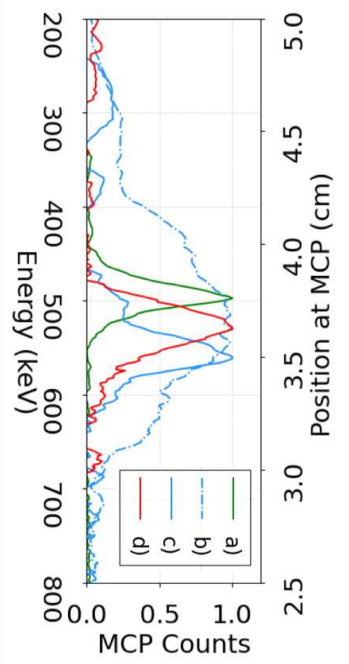

FIG. 7. Example of raw data acquired with the MCP detector coupled to the magnet spectrometer. The total height of the MCP phosphor screen is $7.8 \mathrm{~cm}$. Each image shows the acquisition from an individual shot, with a voltage of $5000 \mathrm{~V}$ and a gain of 20 . The "zero" point that corresponds to the "non deflected" reference position of the proton beam is found by locating the center of the X-ray background along the vertical direction as indicated by the dashed white line. a) Selected proton beam signal obtained with a $20 \mu \mathrm{m}$ entrance slit and a $20 \mu \mathrm{m}$ exit pinhole on the energy selector. b) Selected proton beam signal after passing through the solid carbon foil. c) Selected proton beam signal after passing through the solid carbon foil with a $1 \mathrm{~mm}$ horizontal slit inserted in front of the spectrometer entrance. d) Selected proton beam signal after passing through the WDM sample with a $1 \mathrm{~mm}$ horizontal slit inserted in front of the spectrometer. The X-ray background is produced by the heater beam interaction with the target. e) Vertical lineouts of the images a), b), c), d) as a function of the position on the MCP screen and of the corresponding energy calculated using the energy dispersion by the magnetic field.

beam. The selected pencil-like proton beam undergoes a horizontal energy spread after entering into the magnetic field region. The exit pinhole of $20 \mu \mathrm{m}$ diameter, positioned at $1 \mathrm{~cm}$ after the exit of the magnet, selects a narrow bandwidth of proton beam energies that freely propagates up to the carbon sample. The selector is designed to be fully operational at high repetition rate with a motorization of the dipole magnet moving in and out and a holder for the exit pinhole with horizontal and vertical motorization. The design and the optimization of the energy selector are presented in detail in Ref. ${ }^{41}$. In this work, we selected a proton beam with a central energy of $498 \pm 4 \mathrm{keV}$ and an energy bandwidth of $44 \pm$ $4 \mathrm{keV}$ at FWHM. The energy spectrum of the selected proton beam measured with the high-resolution magnet spectrometer is shown in Fig. 8 and compared with a synthetic spectrum obtained with a FLUKA Monte-Carlo simulation ${ }^{43,44}$ using the experimental selector and detector geometry. The experimental data and the simulated spectrum are in good agreement in their widths at FWHM, while slight differences appear at the wings of the spectra due to proton beam divergence.
Synchronization between the proton and the heater beam

The sub-ns time synchronization was performed for both laser beams at their respective interaction points (proton target and WDM target position) accounting for the time-of-flight (TOF) for $500 \mathrm{keV}$ protons between these points. The proton trajectory was calculated analytically based on the experimental geometry and verified using Monte-Carlo simulations. The heater beam was delayed in respect to the main beam by the proton TOF of $9.2 \mathrm{~ns}$ up to the WDM sample position. This was achieved with the help of a $3 \mathrm{~m}$ long delay line for increasing the heater beam path. This main delay line was coupled with a smaller motorized delay line of $20 \mathrm{~cm}$ length enabling a fine adjustment with a minimum time step of $10 \mathrm{ps}$. The main and the heater beams were synchronized with a $9.2 \mathrm{~ns}$ delay by using photodiodes positioned at their respective interaction points. Both pulse signals were adjusted on a $1 \mathrm{GHz}$ oscilloscope with identical cable lengths using the smaller delay line. The required delay value was obtained with a precision of $\pm 100 \mathrm{ps}$, calculated as $\sigma_{\text {tot }}=\sqrt{\sigma_{\text {stat }}^{2}+\sigma_{p}^{2}}$, where $\sigma_{\text {stat }}=70 \mathrm{ps}$ is a statistical error and $\sigma_{p}=50 \mathrm{ps}$ is the error on the proton TOF calculation. 


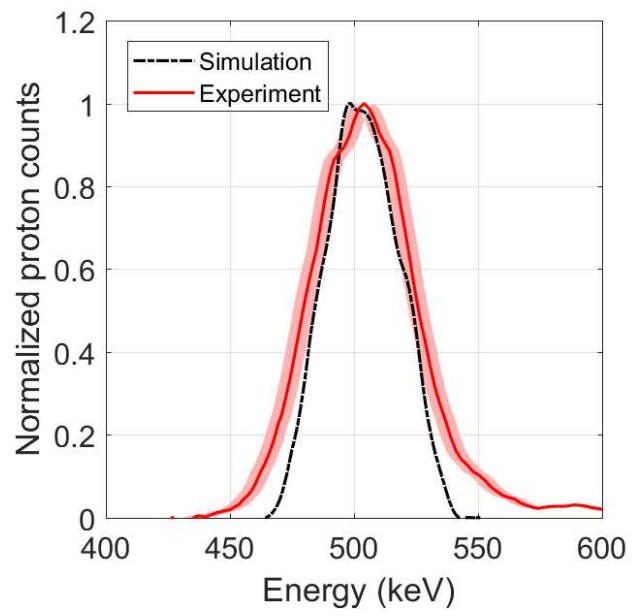

FIG. 8. Selected proton beam spectrum. Comparison between the measured selected proton beam spectrum (red solid curve) and the selected proton beam spectrum obtained from an initial broadband TNSA-like spectrum with energies of $0-2 \mathrm{MeV}$ simulated with the FLUKA Monte-Carlo code using the experimental configuration for the proton energy selector (black dashed curve). The shaded area around the red curve represents the measurement error.

\section{XUV Pinhole Grating (XPHG) diagnostic}

The XPHG diagnostic, based on a a free-standing multi-pinhole X-ray transmission grating, was used to measure the broadband XUV emission from the plasma ${ }^{45,46}$. It had a target view of $30^{\circ}$ in relation to the target normal on the heater beam side. The 500 line $/ \mathrm{mm}$ grating consisted of gold bars of $1 \mu \mathrm{m}$ width and thickness with $1 \mu \mathrm{m}$ openings. It had a larger reinforcement grid structure resulting in an open grating area of $80 \%$, as measured with a scanning electron microscope image. The diffraction efficiency into each of the plus and minus first-order spectra was taken as $1 / \pi^{2}=0.101$, which is the ideal response for such a transmission grating, as was verified by previous authors for this wavelength range ${ }^{69}$. The grating had multiple $400 \mu \mathrm{m}$ pinhole openings and the spectra were obtained from two of the pinholes. The spectra presented are the weighted average of three such single sided spectra per laser shot and averaged over the order of 50 laser shots per experiment. The spectra were filtered by a $400 \mathrm{~nm}$ thick aluminium foil, which transmitted X-rays from $17 \mathrm{~nm}$ to around $70 \mathrm{~nm}$ wavelength, and detected using an Andor iKon-M XUV CCD camera. The distance from the plasma to the pinhole grating was $1197 \mathrm{~mm}$ and the distance from the pinhole to the CCD camera was $99 \mathrm{~mm}$. The diagnostic dispersion was calculated using the distance to the camera, the pixel size and the grating spacing, with an estimated overall accuracy on the order of $\pm 3 \%$. The camera response in counts per keV deposited has been absolutely calibrated with single photons at $5.9 \mathrm{keV}$ energy from an Fe-55 radioisotope X-ray source. The relative response at the XUV wavelengths of interest of $17 \mathrm{~nm}$ to $70 \mathrm{~nm}$ was taken from the manufacturer's published spectral response curve for the camera. Taking the geometric factors, the transmission factors and the response function of the camera into account, absolute emission values were obtained. The estimated accuracy of the absolute emission measurement is on the order of $\pm 20 \%$.

\section{Streaked optical pyrometry (SOP) diagnostic}

The Streaked Optical Pyrometry (SOP) diagnostic was used for measuring the time-resolved black-body temperature of the WDM target with a $10 \mathrm{ps}$ resolution. Due to its sensitivity to low temperatures, this diagnostic is well-suited for measuring the temperature of WDM samples ${ }^{15}$. The SOP diagnostic had a target view of $25^{\circ}$ in relation to the target normal on the heater beam side.

The emission of the WDM target was collected by the optical system, imaging a region of $\sim 400 \mu \mathrm{m}$ onto a Hamamatsu S20 streak camera with a magnification of 5. The interferometric filter was centered at a 532 $\pm 0.6 \mathrm{~nm}$ wavelength with a FWHM bandwidth of $3 \pm$ $0.6 \mathrm{~nm}$ (FI532). An additional color-glass bandpass filter for wavelengths of $360-580 \mathrm{~nm}$ (BG39) was used to mitigate the laser light at $800 \mathrm{~nm}$ wavelength propagating along the collection axis. The wavelength-dependent response of the SOP system within the $3 \mathrm{~nm}$ bandwidth was provided by the manufacturers. The transmission of the optical system for SOP has been measured with a $532 \mathrm{~nm}$ continuous diode laser and the streak camera was absolutely calibrated at the selected wavelength using the calibration of Ref. ${ }^{70}$. The latter was carried out with the same streak camera employed in this experiment and with the filter data set BG38 and FI532 that are similar to the ones we used. The data were acquired inside a time window of $2 \mathrm{~ns}$. The temporal evolution of the temperature was determined using a vertical lineout of the central target region of $50 \mu \mathrm{m}$ diameter corresponding to the proton beam size entering the WDM target. The resulting experimental curve averaged over 65 shots is presented in Fig. 4. The error bar is estimated as $\sigma_{\text {total }}=\sqrt{\sigma_{\mathrm{SE}}^{2}+\sigma_{\text {stat. }}^{2}+\sigma_{\text {calib. }}^{2}}$ that includes the standard error $\sigma_{\mathrm{SE}}=\sigma / \sqrt{N}$ where $\sigma$ is the standard deviation from the mean, a $25 \%$ statistical error $\sigma_{\text {stat. }}$. and a $5 \%$ uncertainty in the detector calibration $\sigma_{\text {calib. }}$.

\section{Modelling}

\section{RALEF2D and MULTI-fs hydrodynamic simulations}

The RALEF2D simulation was performed in axisymmetric geometry using the experimentally measured laser parameters, namely an energy of $0.45 \mathrm{~J}$, a gaussianshaped temporal laser pulse profile with a $217 \mathrm{fs}$ width 
at FWHM and a spatial distribution profile of the focal spot of $\approx 150 \mu \mathrm{m}$ radius.

The density and temperature profiles are sampled with a 5 ps time step for $t=0-100 \mathrm{ps}$ and a $10 \mathrm{ps}$ step for $t$ $=110-500$ ps. The spatial sampling is of $\approx 350$ points for longitudinal rays (along the proton propagation axis) over the target areal density, and of $5 \mu \mathrm{m}$ in the transverse direction up to a radius of $150 \mu \mathrm{m}$.

The MULTI-fs 1D simulation was performed using the same laser energy and pulse duration. In order to represent the radial intensity profile of the focal spot, four separate simulations were performed using the input intensity of the heater calculated within an effective radius that contains $7,20,50,90 \%$ of the total laser energy. The input intensities were used as following: $I_{1}$ $=7.4 \times 10^{15} \mathrm{~W} / \mathrm{cm}^{2}$ at $25 \mu \mathrm{m}$ effective focal spot radius containing $7 \%$ of energy, $I_{2}=5.2 \times 10^{15} \mathrm{~W} / \mathrm{cm}^{2}$ at $50 \mu \mathrm{m}$ focal spot containing $20 \%$ of energy, $I_{3}=1.85$ $\times 10^{15} \mathrm{~W} / \mathrm{cm}^{2}$ at $133.5 \mu \mathrm{m}$ containing $50 \%$ of energy and $I_{4}=7.39 \times 10^{14} \mathrm{~W} / \mathrm{cm}^{2}$ at $283.9 \mu \mathrm{m}$ containing $90 \%$ of energy. For the precision of the calculation of the hydrodynamic parameter values, the target was sampled into 200 layers. The density and temperature profiles were obtained at each layer of the target and sampled with a $10 \mathrm{ps}$ step for $t=0-300 \mathrm{ps}$.

\section{Proton energy-loss calculations}

The energy-loss simulations are performed similarly as in Ref. ${ }^{24}$. The ionization distribution of the plasma is calculated using the collisional-radiative FLYCHK code in local thermal equilibrium ${ }^{50}$, which provides the ion densities $\left(n_{0}, \ldots, n_{6}\right)$ of the different plasma charge states $\left(\mathrm{C}^{0+}, \ldots, \mathrm{C}^{6+}\right)$ for each point of the considered profile. The free electron density is calculated as $n_{e}=6 n_{6}+$ $5 n_{5}+4 n_{4}+3 n_{3}+2 n_{2}+n_{1}$. The mean plasma ionization degree $Z^{*}$ is then determined from the relation $n_{e}=Z^{*} n_{i}$. Here, $n_{i}=\rho N_{A} / A_{t}$ is the total ion density, where $A_{t}=12$ is the molar mass of carbon and $N_{A}$ is the Avogadro number. The free-electron stopping power is calculated using the density $n_{e}$ with the Zimmerman, LiPetrasso and T-Matrix models. The bound-electron stopping power is determined using the ion densities $n_{0}, \ldots, n_{5}$ and the Casas model. The carbon atomic quantities required for the bound electron calculation are taken from Ref. ${ }^{56}$. The total stopping power is obtained as the sum of these contributions:

$$
\frac{d E}{d x}_{\text {total }}=\frac{d E}{d x}_{\text {free }}+\frac{d E}{d x} \text { bound }
$$

The projectile charge state is modeled using the effective charge state predicted by Gus'kov et al. ${ }^{71}$, which is valid in plasma at any projectile velocity. At $500 \mathrm{keV}$ projectile energy, it reaches values $\approx 0.98-0.99$ depending on the target conditions. The projectile slowing down inside the target is taken into account for each step along a)

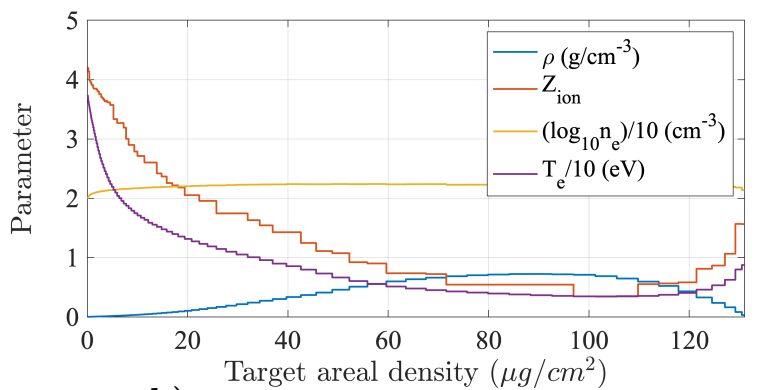

b)

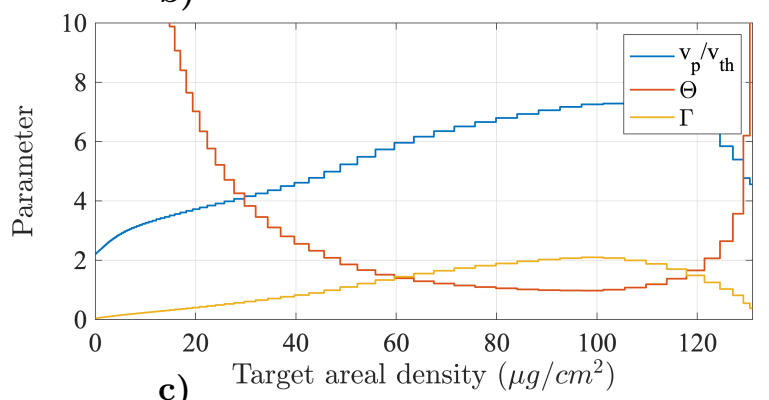

c)

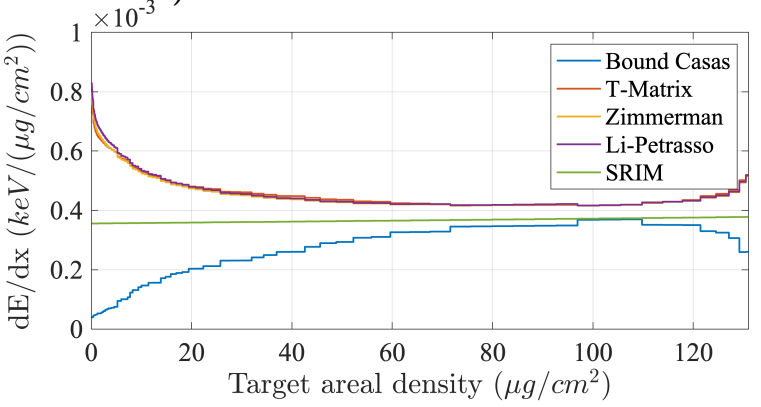

FIG. 9. Plasma conditions and stopping power along the plasma central axis at a time $t=\mathbf{5 0}$ ps. a) Massdensity, electron temperature, free electron density and mean ionization degree. b) Electron coupling $\Gamma$, electron degeneracy $\Theta$ and velocity ratio $v_{p} / v_{t h}$ for a $500 \mathrm{keV}$ energy proton. c) Corresponding stopping-power profiles. The boundelectron contribution as well as the stopping power in the solid target according to the SRIM database are also represented. The $\mathrm{x}$-axis is reported in areal-density units $\left(\mu \mathrm{g} / \mathrm{cm}^{2}\right)$.

the proton propagation path for the beam charge state and the stopping-power calculation. An illustration of stopping-power profile calculation is shown in Fig. 9 for the plasma conditions along the target central axis at $t=$ $50 \mathrm{ps}$ after the beginning of the laser target heating. The target density, temperature, ionization and free electron density profiles are shown in Fig. 9a), the corresponding $\Gamma, \Theta$ and $v_{p} / v_{t h}$ values are shown in Fig. 9b), and the resulting stopping power for one proton of initially $500 \mathrm{keV}$ energy is shown in Fig. 9c). The three ad hoc calculations provide almost identical results, consistently with Fig. 5. The energy loss for one proton at this time 
step is obtained as the integral of the represented stopping power. The energy loss values obtained for all time steps are then convoluted with the spatial and temporal profiles of the probing proton beam. For this purpose, a Monte-Carlo calculation is performed assuming a beam energy bandwidth of $44 \mathrm{keV}$ at FWHM, i.e. a temporal width $\approx 400 \mathrm{ps}$ at FWHM, and a spatial width of $50 \mu \mathrm{m}$ at FWHM. The simulation is performed with the approximate estimated proton number per bunch of 1000 .

\section{TD-DFT stopping-power calculations}

The time-dependent orbital-free density functional theory (TD-OF-DFT) ${ }^{30,31}$ formulation included a nonadiabatic, temperature-dependent kinetic-energy density functional and an exchange-correlation contribution in a local density approximation as well as the usual Hartree and external terms with a local all-electron pseudopotential for carbon. Rectangular prisms of 512 atoms with dimensions $70.0 \times 17.5 \times 17.5 \AA$ were employed as reference cells with the atomic configurations determined from an equilibrium orbital-free molecular dynamics simulation. For a given projectile velocity, the total electron stopping power is determined by the work on the proton as a function of the distance travelled averaged over 2-3 atomic configurations, 10-15 initial positions for the proton, and 3-4 passages of the proton through the cell. At $10 \mathrm{eV}$ temperature, the TD-OF-DFT results have been validated with a full time-dependent KohnSham approach (TD-KS-DFT) utilizing a mixed basis of deterministic and stochastic orbitals ${ }^{67}$, showing an agreement in the stopping power within better than $10 \%$ for projectile velocities larger than $225 \mathrm{keV}$.

\section{$X P H G$ and SOP diagnostic modelling}

For the comparison with the experimental XUV spectra obtained with the XPHG diagnostic, the PrismSPECT atomic code was used to postprocess target pro- files extracted from the RALEF2D and MULTI-fs 1D hydrodynamic simulations. Using the RALEF2D simulation, we considered target profiles at radii $r=30,60$, $90,120,150 \mu \mathrm{m}$ from the proton propagation axis (target center), over the expansion time of $0-300 \mathrm{ps}$, with time steps of $10 \mathrm{ps}$ for $t=0-100 \mathrm{ps}$, and $50 \mathrm{ps}$ for $t=$ 100-300 ps. At each time step, the spatially-integrated emission spectrum was obtained by summing the areaweighted emissivity at each radius. The spectra were then integrated over time to obtain the total space- and time-integrated emission as measured by the XPHG diagnostic on the heater beam side of the target. As for the MULTI-fs simulation, we used target profiles of each of the four simulations with different heater intensities over the expansion time of $0-300 \mathrm{ps}$, with time steps of $10 \mathrm{ps}$ for $t=0-100 \mathrm{ps}$, and $50 \mathrm{ps}$ for $t=100-300 \mathrm{ps}$ to calculate the emissivity with PrismSPECT. For obtaining an area-weighted emissivity at each time-step, a simple model was employed to numerically determine a radius for each average ring of intensity used in simulations and calculate the area.

In order to compare the experimental temperature temporal evolution obtained with Streaked Optical Pyrometry, we considered the RALEF-2D expansion profiles averaged over the central $50 \mu \mathrm{m}$ diameter area and the MULTI-fs expansion profiles of the simulation with the intensity that corresponds to the effective proton probe spot radius of $25 \mu \mathrm{m}$. For this purpose, we considered the temperature with a time step of $10 \mathrm{ps}$ at the critical density $n_{c}=3.88 \times 10^{21} \mathrm{~cm}^{-3}$ that corresponds to the wavelength $\lambda=532 \mathrm{~nm}$.

\section{Data availability}

The data that support the findings of this study are available from the corresponding author upon reasonable request.

\section{REFERENCES}

[1] Hurricane, O. et al. Approaching a burning plasma on the NIF. Phys. Plasmas 26, 052704 (2019).

[2] Zylstra, A. et al. On alpha-particle transport in inertial fusion. Phys. Plasmas 26, 800-806 (2019).

[3] Callahan-Miller, D. A. \& Tabak, M. Progress in target physics and design for heavy ion fusion. Phys. Plasmas 7, 2083-2091 (2000).

[4] Hofmann, I. et al. Review of accelerator driven heavy ion nuclear fusion. Matter Radiat. at Extremes 3, 1-11 (2018).

[5] Roth, M. et al. Fast Ignition by Intense Laser-Accelerated Proton Beams. Phys. Rev. Lett. 86, 436-439 (2001).
[6] Fernández, J. C. et al. Fast ignition with laser-driven proton and ion beams. Nucl. Fusion 54, 054006 (2014).

[7] Kim, J. et al. Self-Consistent Simulation of Transport and Energy Deposition of Intense Laser-Accelerated Proton Beams in Solid-Density Matter. Phys. Rev. Lett. 115, 054801 (2015).

[8] Kim, J. et al. Anomalous material-dependent transport of focused, laser-driven proton beams. Sci. Rep. 8, 17538 (2018).

[9] Sharkov, B. et al. High energy density physics with intense ion beams. Matter Radiat. at Extremes 1, 28-47 (2016). 
[10] Patel, P. et al. Isochoric Heating of Solid-Density Matter with an Ultrafast Proton Beam. Phys. Rev. Lett. 91, 125004 (2003).

[11] McGuffey, C. et al. Focussing Protons from a Kilojoule Laser for Intense Beam Heating using Proximal Target Structures. Sci. Rep. 10, 9415 (2020).

[12] Mancic, A. et al. Picosecond Short-Range Disordering in Isochorically Heated Aluminum at Solid Density. Phys. Rev. Lett. 104, 035002 (2010).

[13] Ping, Y. et al. Heat-release equation of state and thermal conductivity of warm dense carbon by proton differential heating. Phys. Rev. E 100, 043204 (2019).

[14] Grabowski, P. E. et al. Review of the first chargedparticle transport coefficient comparison workshop. High Energy Density Phys. 37, 100905 (2020).

[15] McKelvey, A. et al. Thermal conductivity measurements of proton-heated warm dense aluminum. Sci. Rep. $\mathbf{7}$, 7015 (2017).

[16] White, T. et al. Observation of inhibited electron-ion coupling in strongly heated graphite. Sci. Rep. 2, 889 (2012).

[17] Mackinnon, A. et al. Proton Radiography of a LaserDriven Implosion. Phys. Rev. Lett. 97, 045001 (2006).

[18] Volpe, L. et al. Proton radiography of laser-driven imploding target in cylindrical geometry. Phys. Plasmas 18, 012704 (2011).

[19] Hoffmann, D. H. H. et al. Energy loss of heavy ions in a plasma target. Phys. Rev. A 42, 2313-2321 (1990).

[20] Dietrich, K.-G. et al. Energy loss of heavy ions in a dense hydrogen plasma. Z. Phys. D Atoms Molec. Clust. 16, 229-230 (1990).

[21] Belyaev, G. et al. Measurement of the Coulomb energy loss by fast protons in a plasma target. Phys. Rev. E 53, 2701 (1996).

[22] Frank, A. et al. Energy Loss and Charge Transfer of Argon in a Laser-Generated Carbon Plasma. Phys. Rev. Lett. 110, 115001 (2013).

[23] Gericke, D. O. \& Schlanges, M. Energy deposition of heavy ions in the regime of strong beam-plasma correlations. Phys. Rev. E 67, 037401 (2003).

[24] Cayzac, W. et al. Predictions for the energy loss of light ions in laser-generated plasmas at low and medium velocities. Phys. Rev. E 92, 053109 (2015).

[25] Cayzac, W. et al. Experimental discrimination of ion stopping models near the Bragg peak in highly ionized matter. Nat. Commun. 8, 15693 (2017).

[26] Frenje, J. A. et al. Experimental Validation of Low-Z Ion-Stopping Formalisms around the Bragg Peak in HighEnergy-Density Plasmas. Phys. Rev. Lett. 122, 015002 (2019).

[27] Gericke, D. O. et al. Stopping power of nonideal, partially ionized plasmas. Phys. Rev. E 65, 036406 (2002).

[28] Edie, D. J. et al. $\alpha$-particle stopping and electron-ion energy relaxation in highly compressed ICF fuel. In Eur. Phys. J. Web Conferences, vol. 59, 5018 (2013).

[29] Deutsch, C. et al. Multiple scattering in electron fluid and energy loss in multi-ionic targets. Nucl. Instrum. Methods Phys. Res. A 733, 39-44 (2014).

[30] Ding, Y. H. et al. Ab Initio Studies on the Stopping Power of Warm Dense Matter with Time-Dependent Orbital-Free Density Functional Theory. Phys. Rev. Lett. 121, 145001 (2018).

[31] White, A. J. et al. Time-dependent orbital-free density functional theory for electronic stopping power: Compar- ison to the Mermin-Kohn-Sham theory at high temperatures. Phys. Rev. B 98, 144302 (2018).

[32] Hayes, A. C. et al. Reaction-in-flight neutrons as a test of stopping power in degenerate plasmas. Phys. Plasmas 22, 082703 (2015).

[33] Hayes, A. C. et al. Plasma stopping-power measurements reveal transition from non-degenerate to degenerate plasmas. Nat. Phys. 16, 432-437 (2020).

[34] Zylstra, A. B. et al. Measurement of charged-particle stopping in warm dense plasma. Phys. Rev. Lett. 114, 215002 (2015).

[35] Chen, S. N. et al. Experimental evidence for the enhanced and reduced stopping regimes for protons propagating through hot plasmas. Sci. Rep. 8, 14586 (2018).

[36] Ren, J. et al. Observation of a high degree of stopping for laser-accelerated intense proton beams in dense ionized matter. Nat. Commun. 11, 5157 (2020).

[37] Zylstra, A. B. et al. Platform development for $\mathrm{dE} / \mathrm{dx}$ measurements on short-pulse laser facilities. High Energy Density Phys. 35, 100731 (2020).

[38] Jahn, D. et al. Focusing of multi-MeV, subnanosecond proton bunches from a laser-driven source. Phys. Rev. Accel. Beams 22, 011301 (2019).

[39] Volpe, L., Fedosejevs, R., Gatti, G., Pérez-Hernández, J. A., Méndez, C., Apiñaniz,J., Vaisseau,X., Salgado, C., Huault, M. Malko, S. Zeraouli, G. Ospina, V. Longman, A. De Luis, D. Li, K. Varela, O. García, E. Hernández, I. Pisonero, J. García Ajates, J. Alvarez, J. García, C. Rico, M. Arana, D. Hernández-Toro, J. \& Roso, L. Generation of high energy laser-driven electron and proton sources with the 200 TW system VEGA 2 at the Centro de Laseres Pulsados. High Power Laser Sci. Eng. 7, 25 (2019).

[40] Wilks, S., Langdon, A., Cowan, T., Roth, M., Singh, M., Hatchett, S., Key,M. H., Pennington, D., MacKinnon, A. \& Snavely, R. Energetic proton generation in ultraintense laser-solid interactions. Phys. Plasmas 8, 542 (2001).

[41] Apiñaniz, J., Malko, S., Fedosejevs, R., Cayzac, W., Vaisseau, X., De Luis, D., Gatti, G., McGuffey, C., Bailly-Grandvaux, M., Bhutwala, K., Ospina-Bohorquez, V., Balboa, J., Santos, J. J., Batani, D., Beg, F., Roso, L., Perez-Hernandez, J. A. \& Volpe, L. A quasi-monoenergetic short time duration compact proton source for probing high energy density states of matter. Sci. Rep. 11,6881 (2021).

[42] Prasad, R. et al. Thomson spectrometer-microchannel plate assembly calibration for $\mathrm{MeV}$-range positive and negative ions, and neutral atoms. Rev. Sci. Instrum. 84, 053302 (2013).

[43] Ferrari, A., Sala, P., Fasso, A., \& Ranft, J. FLUKA: a multi-particle transport code. CERN-200510 INFN/TC_05/11, SLAC-R-773, (2005).

[44] Böhlen, T., Cerutti, F., Chin, M., Fasso, A., Ferrari, A., Ortega, P., Mairani, A., Sala, P., Smirnov, G. \& Vlachoudis, V. The FLUKA Code: Developments and Challenges for High Energy and Medical Applications. Nuclear Data Sheets 120, 211-214 (2014).

[45] Ceglio, N., Kauffman, R., Hawryluk, A., \& Medecki, H. Time-resolved x-ray transmission grating spectrometer for studying laser-produced plasmas. Appl. Optics 22, 318-327 (1983).

[46] Eidmann, K., Kishimoto, T., Herrmann, P., Mizui, J., Pakula, R., Sigel, R., \& Witkowski, S. Absolute soft x-ray 
measurements with a transmission grating spectrometer. Laser Part. Beams 4, 521-536 (1986).

[47] Tauschwitz, A. et al. 2D radiation-hydrodynamics modeling of laser-plasma targets for ion stopping measurements. High Energy Density Phys. 9, 158-166 (2013).

[48] Faik, S. et al. Creation of a homogeneous plasma column by means of hohlraum radiation for ion-stopping measurements. High Energy Density Phys. 10, 47-55 (2014).

[49] Basko, M. M. et al. One-dimensional study of the radiation-dominated implosion of a cylindrical tungsten plasma column. Plasma Phys. Control. Fusion 54, 055003(1-16) (2012).

[50] Chung, H.-K., Chen, M. H., Morgan, W. L., Ralchenko, Y. \& Lee, R. W. FLYCHK: Generalized population kinetics and spectral model for rapid spectroscopic analysis for all elements. High Energy Density Phys. 1, 3-12 (2005).

[51] Ramis, R., Eidmann, K., Meyer-ter-Vehn, J., Hüller, S. MULTI-fs - A computer code for laser-plasma interaction in the femtosecond regime. Comput. Phys. Commun. 183, 637-655 (2012).

[52] MacFarlane, J. J., Golovkin, I. E., Woodru, P. R., Welch, D. R, Oliver, B. V., Melhorn, T. A., \& Campbell, R. B., A. Hammel, D. D. Meyerhofer, J. Meyer ter Vehn, and H. Azechi 2, (2004).

[53] P. C. Sciences. http://www.prism-cs.com/

[54] Ziegler, J. F., Biersack, J. P. \& Littmark, U. The Stopping and Range of Ions in Matter (Pergamon, New York, 1985).

[55] Melhorn, T. et al. A finite material temperature model for ion energy deposition in ion-driven inertial confinement fusion targets. J. Appl. Phys. 52, 6522-6532 (1981).

[56] Casas, D. et al. Evaluation of slowing down of proton and deuteron beams in $\mathrm{CH} 2, \mathrm{LiH}$, and $\mathrm{Al}$ partially ionized plasmas. Phys. Rev. E 88, 033102 (2013).

[57] Casas, D. et al. Stopping power of a heterogeneous warm dense matter. Laser Part. Beams 34, 306-314 (2016).

[58] Zylstra, A. et al. Modified parameterization of the LiPetrasso charged-particle stopping power theory. Phys. Plasmas 16, 122703 (2019).

[59] Brown, L. S., Preston, D. L. \& Singleton, R. L., Jr. Charged particle motion in a highly ionized plasma. Phys. Rep. 410, 237-333 (2005).

[60] Gericke, D. O. Stopping power for strong beam-plasma coupling. Laser Part. Beams 20, 471-474 (2002).

[61] Gericke, D. O. \& Schlanges, M. Beam-plasma coupling effects on the stopping power of dense plasmas. Phys. Rev. E 60, 904-910 (1999).

[62] Zimmerman, G. LLNL, Report No. UCRL-JC-105616. (1990).

[63] Maynard, G. and Deutsch, C. Born random phase approximation for ion stopping in an arbitrarily degenerate electron fluid. J. Phys. 46, 1113-1122 (1985).

[64] Faussurier, G. et al. Equation of state, transport coefficients, and stopping power of dense plasmas from the average-atom model self-consistent approach for astrophysical and laboratory plasmas. Phys. Plasmas 17, 052707 (2010).

[65] Gauthier, M. et al. Stopping power modeling in warm and hot dense matter. High Energy Density Phys. 9, 488-495 (2013).

[66] Faussurier, G. \& Blancard, C. Friedel sum rule at finite temperature in hot dense plasmas. Phys. Plasmas 28, 042710 (2021).
[67] White, A. J. \& Collins, L. A. Fast and Universal KohnSham Density Functional Theory Algorithm for Warm Dense Matter to Hot Dense Plasma. Phys. Rev. Lett. 125, 055002 (2020).

[68] Lommel, B., Hartmann, W., Kindler, B., Klemm, J. \& Steiner, J. Preparation of self-supporting carbon thin films. Nucl. Instrum. Methods Phys. Res. A 480, 199203 (2002).

[69] Eidmann, K., Kuhne, M., Moller, P., \& Tsakiris, G., Characterization of pinhole transmission gratings. J. $X$ Ray Sci. Technol. 2, 259-273 (1990).

[70] Vauzour, B. Etude expérimentale du transport d'électrons rapides dans le cadre de l'allumage rapide pour la fusion inertielle. PhD Thesis, University of Bordeaux (2012).

[71] Gus'kov, S. Y. et al. A method for calculating the effective charge of ions decelerated in a hot dense plasma. Plasma Phys. Rep. 35, 709-718 (2009).

\section{ACKNOWLEDGEMENTS}

We gratefully thank the director Luis Roso and the team of the VEGA II laser facility at Centro de Laseres Pulsados for their expert support in performing the experiment, as well as B. Lommel and the team of the GSI Target Laboratory for the preparation and the delivery of the sample targets. The research leading to these results has received funding from LASERLAB-EUROPE V (Grant Agreement No. 871124, European Union Horizon 2020 research and innovation program) and from IMPULSE (Grant Agreement No. 871161, European Union Horizon 2020 research and innovation program). Support from Spanish Ministerio de Ciencia, Innovación y Universidades through the PALMA Grant No. FIS201681056-R, ICTS Equipment Grant No. EQC2018-005230P, from LaserLab Europe IV Grant No. 654148, from Junta de Castilla y León Grants No. CLP087U16 and No. CLP263P20, from the Canadian Natural Sciences and Engineering Research Council (RGPIN-2019-05013) and from the LIGHT S\&T Graduate Program (PIA3 Investment for the Future Program, ANR-17-EURE-0027) are acknowledged. The US collaborators acknowledge funding from the National Nuclear Security Administration (Grant DE-NA0003876 HEDLP). A. J. W and L. A. C acknowledge support under Science Campaign 4 by the U.S Department of Energy through the Los Alamos National Laboratory, operated by Triad National Security, LLC for the National Nuclear Security Agency of the U.S Department of Energy (Contract No. 89233218NCA000001). Research presented in this article was also supported by the Laboratory Directed Research and Development program of Los Alamos National Laboratory under project number 20210233ER. S. X. H acknowledges the support by the Department of Energy National Nuclear Security Administration under Award Number DE-NA0003856 and U.S National Science Foundation PHY Grant No. 1802964. 


\section{AUTHOR CONTRIBUTIONS}

L. V, S. M and W. C conceived the idea, designed the experiment and wrote the paper. L. V and S. M led the experiment. S. M coordinated the analysis of the experimental results and of the simulations. W. C coordinated the stopping-power calculations and performed the energy-loss simulations. J. V provided support for the RPA stopping-power calculations. G. F performed the QAAM stopping-power calculations. L. A. C, A. J. W and S. X. H provided theoretical support and performed the TD-DFT stopping-power calculations. A. T performed the RALEF2D hydrodynamic simulation. S. M, L. V, W. C, R. F, J. A. P, J. I. A, V. O, K. B, M. B, X. V, C. M, M. H, P. N, G. P and $\mathrm{C} . \mathrm{V}$ participated to the experimental campaign and the preparation campaign. S. M, J. I. A and L. V operated the proton detector diagnostic. S. M, C. M and X. V analyzed the proton diagnostic data.
R. F and K. B operated the XPHG spectrometer and analyzed the XPHG measurements. V. O operated the SOP diagnostic. V. O and M. B analyzed the SOP data. S. M performed the modelling and the simulations for both plasma emission diagnostics. D. L, J. I. A and G. G built the magnetic selector. R. F, J. S, D. B, J. V and F. N. B provided theoretical support.

\section{ADDITIONAL INFORMATION}

Competing financial interests: The authors declare no competing financial interests.

$\begin{array}{cccr}\begin{array}{c}\text { Reprints } \\ \text { formation }\end{array} \text { is } & \text { and available } & \text { online } & \text { at }\end{array}$

http://npg.nature.com/reprintsandpermissions/

Correspondence and requests for materials should be addressed to S.M. 